\title{
ECONOMÍAS AISLADAS, PEQUEÑAS Y DEPENDIENTES DE COMMODITIES: EL CASO DEL PETRÓLEO Y METANOL EN LA COMUNA DE PUNTA ARENAS, CHILE.
}

\author{
SERGIO SOZA-AMIGO* Y PATRICIO AROCA*
}

\begin{abstract}
RESUMEN ${ }^{1,2}$
Punta Arenas es una comuna ubicada en el extremo sur de Chile, cuya economía se basa en la producción de dos materias primas (commodities): petróleo y metanol. En este contexto, el objetivo principal de este artículo es estimar el impacto de estas materias primas sobre la producción, el ingreso y el empleo de la comuna, en donde la importancia relativa de la producción de estas materias primas ha estado cambiando del petróleo al metanol.

Usando una matriz input-ouput, demostramos que, los encadenamientos de estas materias primas, en economías que son pequeñas y aisladas generan un bajo impacto en la producción e ingreso. Adicionalmente, se detecta que mientras los encadenamientos de empleo son grandes, se observa que el nivel del mismo en estas industrias es muy bajo respecto al total de empleados de Punta Arenas.
\end{abstract}

PALABRAS CLAVE: Commodities, multiplicadores, elasticidades, input- output y desarrollo local.

Clasificación JEL

C67; D57; O10; R15 y R34

* Profesor Asociado, Facultad de Ciencias Económicas y Jurídicas, Universidad de Magallanes, Punta Arenas, Chile, e-mail: sergio.soza@umag.cl.

* Profesor Titular, Departamento de Economía, Director del Instituto de Economía Aplicada Regional (IDEAR), Universidad Católica del Norte, Antofagasta, Chile, e-mail: paroca@ucn.cl.

1 Se reconoce y agradece el apoyo del Núcleo de la Iniciativa Científica Milenio "Ciencia Regional y Políticas Públicas".

2 Una versión preliminar de este trabajo se presento en la XIII Reunión de Economía Mundial y en las IV Jornadas Españolas de Análisis Input-Output. 


\title{
SMALL, ISOLATED AND COMMODITIES DEPENDENT ECONOMIES: THE CASE OF THE OIL AND METHANOL IN THE COUNTY OF PUNTA ARENAS, CHILE.
}

\begin{abstract}
Punta Arenas is a county in the Chilean extreme south which economy is driven by the production of two commodities: oil and methanol. In this context, the main aim of this paper is to estimate the impact of these commodities on output, income and employment of the county, where the relative significance of the commodities production has been changing from oil to methanol.

Using an input-output framework, we show that, the linkages and the impact of these commodities in this small and isolated economy in output and income. While in employment their linkages are large, the level of the employment on this industry is very low compare with the Punta Arenas total employees.
\end{abstract}

KEY WORDS: Commodities, multipliers, elasticity, input-output and local development.

JEL Classification

C67; D57; O10; R15 y R34.

\section{INTRODUCCIÓN}

Estudiar los impactos de la producción de "commodities" sobre las economías locales es complejo e interesante en varios sentidos, pero en particular y para los fines de este trabajo, en dos. Primero, dado que el impacto puede tomar distintas dimensiones, los múltiples efectos no necesariamente son los esperados; $y$, en segundo lugar, por las consecuencias que se presentan cuando se gestan en ellas alteraciones productivas que terminan repercutiendo en sus respectivas economías.

Por esto, se hace relevante para el analista, comprender cómo repercuten tales actividades; ya que, históricamente ellas han sido asociadas a una generación de bajos efectos sobre el bienestar de los habitantes locales, pero altos crecimientos en la producción de bienes y servicios (Aroca (2001), Miller (1957) e Isard y Kuenne (1953)).

En economías pequeñas que se encuentran en vías de desarrollo; o, al decir de Krugman y Obstfeld (2006: 259), menos desarrolladas, el fuerte deseo por determinar y encontrar una estructura económica que permita un rápido desarrollo; en un entorno con insuficiente disponibilidad de recursos, y en especial en economía aisladas, puede llevar a centrar los esfuerzos en el crecimiento, sin considerar el impacto que este puede tener sobre la calidad de los individuos que habitan la zona.
Utilizando la comuna de Punta Arenas y siguiendo una metodología de evaluación de impacto basado en modelos de insumo-producto se consideran dos escenarios, una economía abierta y otra cerrada. En la primera, se asumen que los salarios se gastan fuera de la región, mientras que en la segunda los salarios pagados en la comuna se gastan íntegramente en la región, utilizando para ello la matriz de insumo-producto recientemente construida para la comuna de Punta Arenas ${ }^{3}$.

Se ha optado por la Comuna de Punta Arenas, por las siguientes razones:

1. La comuna de Punta Arenas, se ubica en el sur austral de Chile en la región de Magallanes, está inserta geográficamente en la zona sub Antártica, por tanto, es una zona que por naturaleza es extrema y, aislada geográficamente, lo que permite separar ciertos efectos e interrelaciones de mejor manera.

2. La comuna basa su actividad económica en, la agricultura, silvicultura, turismo; $y$, en especial en los commodities (petróleo y gas natural), con sus respectivos derivados (combustibles y metanol).

3. Dada la condición geográfica de la comuna -prácticamente una total aislación-, se favorecen los análisis que guardan relación con, simulaciones, la cuantificación de efectos; y, la separación de las

3 Soza- Amigo, S., J. Fernández y H. Rocha (2010): Matriz Insumo- Producto para la Comuna de Punta Arenas. 
interrelaciones que interesan estudiar, en especial, las que se vinculan con el intercambio regional.

En base a los antecedentes antes expuestos, se plantean interrogantes que dicen relación al menos en dos ámbitos; por un lado, los referidos al desarrollo de la comuna, con los consiguientes efectos e impactos de los commodities en ella; $y$, por otro, a la experiencia que se puede traspasar a otras economías, en aspectos tales como; la influencia que ejercen los commodities en economías que son pequeñas y están aisladas geográficamente -lo que se puede extrapolar a economías mayores y no necesariamente aisladas, pero que sí cuentan con estos productos-; $y$, las que dadas determinadas condiciones, e.g., el estar insertas en áreas de flujo de capitales importantes, no pueden evaluar en detalle las repercusiones que se originan por la explotación de estos productos (por ejemplo, el caso del cobre en el norte de Chile; para comprender mejor éste aspecto se sugiere revisar Aroca 2001 y Aroca y Atienza 2011).

En relación al primer aspecto, es válido preguntarse, ¿cómo influir para que la producción de la región; $y$, en especial en la comuna más importante de Magallanes se incremente de manera sostenida; o, al menos, se mantenga en el tiempo?, ¿de qué manera se puede aumentar la participación en la producción nacional?, ¿cuáles son los elementos o factores que afectan el crecimiento sostenido de la producción y cómo se relacionan entre ellos?, ¿cuáles son las actividades económicas comunales más relevantes que empujan su crecimiento y que son claves para el incremento de la producción futura?

Lo anterior, se extiende al agregar las interrogantes que se vinculan a los commodities, por ejemplo, ¿qué impacto real, tienen los commodities en la generación y división del trabajo por unidad de producto?, ¿en la generación de empleos, directos $e$ indirectos?, ¿afectan sustancialmente en el consumo los salarios y rentas que se obtienen a partir de los commodities que se explotan localmente?; $y$, por último, es válido preguntarse, ¿qué hacer para que los commodities que se explotan en las distintas economías, contribuyan de mejor manera a su desarrollo?

El trabajo, luego de presentar los objetivos e hipótesis, se estructura de la siguiente manera: 1) se realiza una breve descripción de la región de
Magallanes, considerando sus condiciones geográficas y económicas (desde 1960 al año 2006); 2) basados en dos escenarios (abierto se gastan fuera de la región los pagos por los factores productivos e impuestos y, cerrado en donde se asume que todo se gasta en la comuna), se determinan y analizan distintos multiplicadores para la comuna de Punta Arenas (de producto, ingreso y empleo), centrando el análisis en los commodities materia de estudio; 3) se determinan distintos encadenamientos, basados en las propuestas de Rasmmusen (1956) y de descomposición de Soza-Amigo (2007), para lo cual se consideran distintos efectos (total, directo e indirecto y un efecto parcializado); 4) Dado que la matriz es referida al año 2003, y que el último CENSO realizado en Chile data para el año 2002, se cruzan ambos datos y se analiza los efectos de la conmutación en la comuna. Finalmente, se discute sobre la metodología empleada y algunos resultados obtenidos, para terminar presentando algunas conclusiones que dan cuanta del impacto que tienen los commodities en la comuna de Punta Arenas, en términos de efectos multiplicativos sobre, la producción, ingreso y empleo, además, se destacan los principales hallazgos referidos a los impactos directos e indirectos que se dan a partir de estos commodities y, para cerrar ésta parte, se destacan los efectos en términos de beneficios que se originan por la conmutación originados por éstos productos. Conclusiones que por cierto, pueden ser extrapolables a otras economías dado que aportan a la comprensión del desarrollo local y, en especial, de los commodities.

\section{OBJETIVOS}

Evaluar los impactos que generan en una economía pequeña y aislada geográficamente, dos commodities (petróleo y metanol), considerando para tales propósitos dos escenarios, que la economía ésta abierta y, otro en donde se asume que ella ésta cerrada.

\section{OBJETIVOS ESPECÍFICOS}

O1: $\quad$ Estudiar los efectos multiplicativos de los commodities, en la producción, ingreso y empleo.

O2: Analizar los efectos directos e indirectos que generan los commodities. 
O3: $\quad$ Evaluar el impacto de la conmutación en los commodities estudiados.

O4: $\quad$ Cotejar los efectos de los commodities versus el resto de productos o servicios.

\section{HIPÓTESIS}

H1: $\quad$ Los commodities presentan una importante participación en la producción (PIB), pero no aportan significativamente al desarrollo local.

H2: $\quad$ Altos multiplicadores para el ingreso o empleo, no implican grandes encadenamientos (hacia atrás y adelante).

\section{LA REGIÓN DE MAGALLANES Y LA COMUNA DE PUNTA ARENAS}

La zona donde está inserta la comuna cuenta con una importante cantidad de praderas, bosques, fiordos, golfos, canales, islas y archipiélagos. Tiene 7 millones de hectáreas bajo el Sistema Nacional de Áreas Protegidas, entre los que se encuentran atractivos como el Parque Nacional Torres del Paine, el Cabo de Hornos y el Campo de Hielo. A lo que se debe agregar otros lugares de interés como el estrecho de Magallanes, la Antártica Chilena, y el paso natural entre los océanos Atlántico y Pacifico y el mar de Drake.
Con el fin de ilustrar económicamente el escenario elegido, se presenta la evolución del Producto Interno Bruto (PIB) regional en contraste al nacional, en tal sentido, se puede observar que si bien la tendencia en el tiempo ha sido la de un PIB creciente para la región, también se identifican importantes variaciones negativas, las cuales comparativamente no son similares a la tendencia nacional (ver Fig. 1). Las variaciones, en especial las negativas, del PIB de la región han sido más agudas que las registradas para el país. Por su parte, en términos de la participación del PIB de la región respecto al PIB nacional, ésta equivale a un poco más del $1 \%$ del total, la cual no ha variado de manera significativa en el tiempo.

La evolución del PIB muestra que la región tuvo un ciclo de alto nivel de crecimiento en el periodo que va desde 1975 hasta 1982, exactamente entre las dos más fuertes crisis que ha tenido Chile en los últimos 50 años, la crisis del petróleo y la del endeudamiento. Para una región que es la única que tiene explotación de petróleo, era esperable que este periodo fuese positivo, a diferencia del resto del país, dado el inmenso salto del precio del petróleo en la época, pasando de 2.8 dólares el barril en 1973 a más de 30 dólares en 1974.

Sin embargo, la crisis del endeudamiento, al igual que en todo el país tuvo un fuerte impacto negativo, del cual toma mucho más tiempo a la

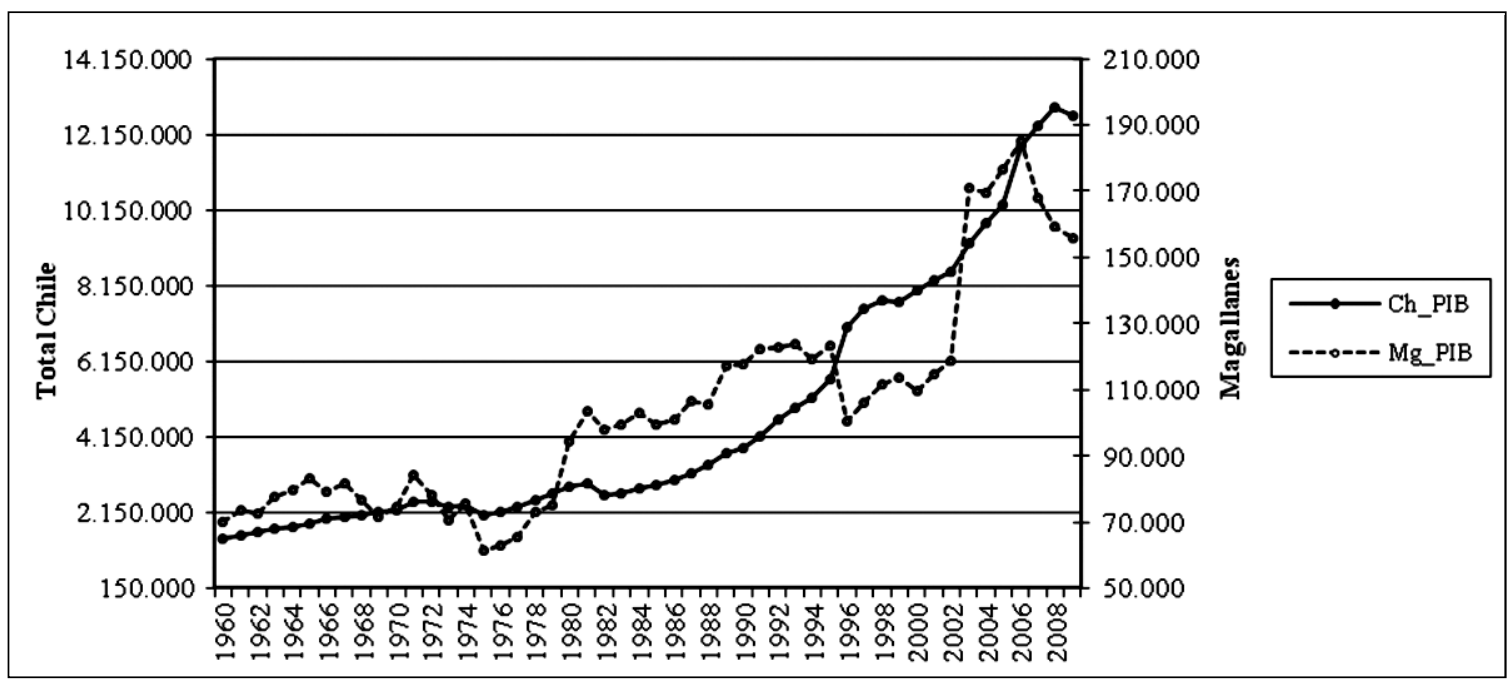

Fig. 1. PIB para Chile y Magallanes (1960- 2009, millones pesos de 1986). Fuente: Soza- Amigo (2010), basado en MIDEPLAN y Banco Central. 


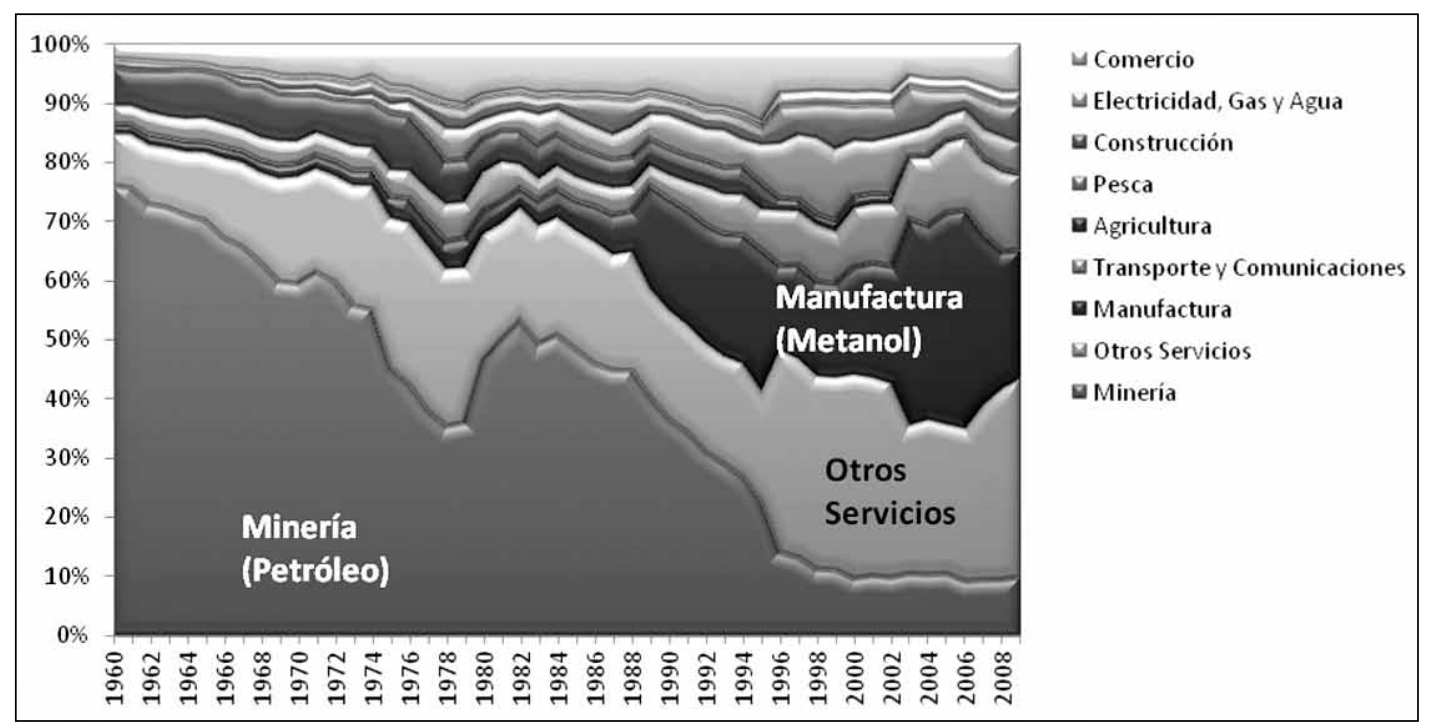

Fig. 2. Evolución de la participación sectorial de Magallanes (1960- 2009).

Fuente: MIDEPLAN y Banco Central de Chile.

región de Magallanes recuperarse, comparada con el resto del país. Durante los siguientes 15 años, la región de Magallanes logra crecer, sin embargo, lo hace a una velocidad muy inferior a la de la nación y la crisis asiática de 1997-1998, hace regresar a Magallanes a niveles de producción similares a los alcanzados en la crisis de la deuda.

Afortunadamente, desde ahí en adelante, retoma un nivel de crecimiento muy superior al resto del país y para mediados de la década de 2000, ya ha alcanzado los niveles de crecimiento de la nación.

Al revisar la historia del PIB regional, se observa que a principios de los años 60, la actividad económica se centraba en un 75\% en la minería, la cual en los últimos años ha ido perdiendo relevancia, cayendo a un escaso 10\%. En los inicios de los años 80 , comienzan a tener mayor participación las actividades ligadas a la industria manufacturera (3\%) y servicios (15\%), llegando en el año 2009 a tener una participación de alrededor de 40 y 30\% respectivamente. En referencia al sector agrícola y pesca, se observa que el primero pierde importancia, pasando desde un $8 \%$ en los años 60 a prácticamente cero en el 2009, mientras que en la pesca, las cifras se invierten.

Desde una perspectiva más global, se observa que la economía en los últimos años presenta una diversificación importante respecto a lo concentrada que era en los años 60 (minería). Tal diversificación se justificaría por la evolución que ha significado pasar desde actividades primarias (extracción de petróleo y carbón), a secundarias, motivadas por el metanol en especial desde principios de los $90 \mathrm{y}$, de servicios en la actualidad (actividades vinculadas al petróleo, gas natural, y comercio motivado por el turismo).

En lo referente a la importancia que tiene la comuna de Punta Arenas en el contexto regional, se puede señalar que según la Matriz Insumo-Producto de la comuna para el año 2003, se determinó que su Producto Interno Bruto fue de $\mathrm{M} \$ 832.502$. En tal sentido, y sólo como referencia, se puede cotejar con el PIB de la Región para ese año, el que de acuerdo al Banco Central de Chile, fue de $\mathrm{M} \$ 862.907$, aun cuando se es consciente que los procedimiento para la obtención de ambas magnitudes no son iguales, ellas si nos dan una referencia en términos de lo importante que es PIB de tal comuna para el contexto regional.

En otro ámbito, del Servicios de Impuestos Internos (ver Fig. 3), se puede observar que las ventas totales de la Comuna de Punta Arenas, desde el año 1999 al 2003, promediaron en total algo más de un $92 \%$, cifra que se mantiene, en general, para todas las actividades, con la sola excepción de agricultura que para esos años promedia aproximadamente un $82 \%$, estando todas las demás actividades, en general, por sobre el 90\%. 


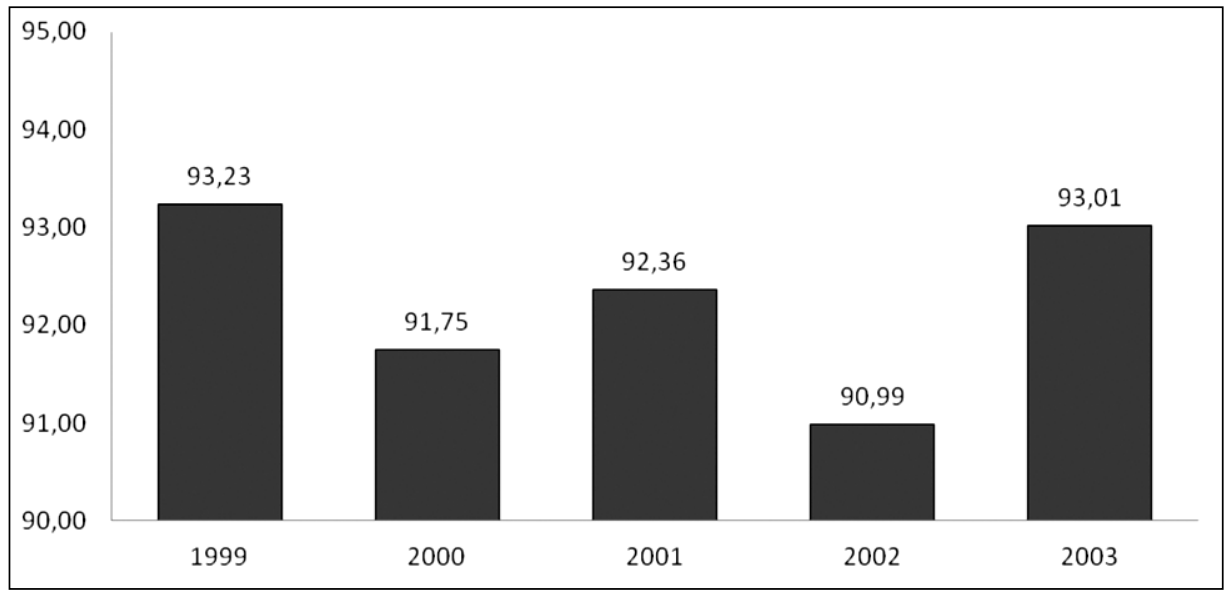

Fig. 3. Porcentaje de Ventas de la Comuna de P. Arenas en Magallanes. Fuente: Propia en base a SII.

En una línea similar a lo ya expuesto, se rescata del CENSO del año 2002, que del total de personas que trabajaban en la región por un ingreso (56.971 personas), un 77\% (43.722 personas) se encontraba en la Comuna de Punta Arenas, lo que nuevamente confirma lo importante que es ella para el contexto regional.

\section{METODOLOGÍA}

Uno de los aportes más importantes del famoso economista inglés Sir John Maynard Keynes (1936) fue la idea del multiplicador. La intuición básica de este concepto consiste en que la economía crece producto de la interacción entre los sectores. Es decir, un cambio en la demanda de un sector, por ejemplo, el Gobierno decide construir una nueva carretera en la Región de Magallanes, tendrá un efecto multiplicador a través de la demanda de insumos necesarios para construir la carretera. Luego, las empresas que ven incrementada su demanda, deberán contratar insumos para hacer frente a este aumento, contratando insumos desde otros sectores y así sucesivamente continuará este proceso multiplicador de la economía.

La idea de Keynes, fue pensada a nivel macroeconómico, es decir, un proceso que ocurre en la economía como un todo, y este concepto fue clave para sustentar la política que saco al mundo de la recesión de 1929, donde su recomendación principal fue aumentar el gasto del gobierno en algún sector para reemplazar el gasto privado que estaba muy deprimido y que el efecto multiplicador se encargaría de llevar el impulso a todo el resto de la economía.

El economista alemán y educado en Rusia, Wassily Leontief (1941) propuso una forma concreta de medir estos multiplicadores entre sectores, a través del método de insumo-producto, por lo cual fue galardonado con el premio Nobel de Economía en 1973. En su libro, Leontief presenta un estudio de la estructura de la economía de Estados Unidos, describiendo extensamente cuales eran los sectores más encadenados o con mayor impacto en la economía y por lo tanto más importantes para hacer política de reactivación.

El economista japonés Kenichi Miyazawa (1976) extiende la idea de Keynes y Leontief al proponer que no sólo los sectores productivos tienen un efecto multiplicador, sino que también lo tienen los dueños de los factores productivos: capitalistas y trabajadores al gastar el ingreso que obtienen producto de la venta de estos. La literatura reciente pone especial énfasis en el impacto del gasto del ingresos de los trabajadores sobre la economía, que muchas veces resulta más significativo que el impacto del gasto que hacen la empresas del sector, especialmente cuando el análisis de realiza a nivel comunal o regional.

Recientemente, la idea de analizar los multiplicadores se ha desarrollado extensamente y tres buenas síntesis pueden encontrarse en Hewings 
(1985), Isard et al (1998), Miller and Blair (2009) y una reciente aplicación para la Región de Magallanes en Soza- Amigo y Aroca (2010).

En este contexto, los multiplicadores son utilizados por señalar cuánto se produce en una economía, por cada unidad que es consumida, invertida o exportada; lo que permite indagar sobre las posibles repercusiones que tienen las modificaciones que se pueden dar en una actividad. En especial, en los commodities, es importante e ilustrativo, sobre todo si se establecen, por ejemplo, escenarios como los elegidos; una economía pequeña, aislada geográficamente que depende de ciertos commodities y que se analiza bajo dos supuestos importantes (sistema productivo abierta y cerrada).

El sistema cerrado, propuesto por Miyazawa (op.cit), extiende la visión tradicional (sistema abierto) vinculando las interrelaciones intersectoriales con los receptores de los pagos de los factores productivos, especialmente con el gasto que realizan los trabajadores, ya que ellos pueden asociarse más genuinamente al territorio, no así los pagos al factor capital, que muchas veces dejan el territorio donde se realizan.

Analizar los escenarios anteriores obedece a que, cuando se trabaja con un sistema abierto, se asume implícitamente que los trabajadores y dueños del capital gastan sus ingresos fuera de la economía.

Esto puede ser plausible para los dueños del capital y el gobierno, porque los primeros no viven en la economía que les permite obtener sus rentas y el gobierno tiene un presupuesto centralizado, luego, es dable esperar que el gasto de ellos se realice en otras zonas.

Por otra parte, si ciertas actividades descansan en sistemas de turno, es razonable pensar que tales trabajadores no vivan necesariamente en donde laboran -efecto conmutación-, esto es, trabajan en un área pero viven en otra, dado que el sistema de turnos los obliga a permanecer en sus lugares de trabajo, pero no a vivir en la misma zona.

Aun cuando lo anterior, es posible que ocurra, tales situaciones no siempre se dan con la mayoría de los trabajadores, dado que; en general, laboran y viven en la misma economía en la cual perciben sus respectivos salarios -en especial, si está se encuentra aislada geográficamente-, por lo que es más real asumir que sus rentas se gastan en la misma economía y de acuerdo a la estructura de consumo que en ella se observa (sistema cerrado; Aroca 2001 y Soza-Amigo y Aroca 2010).

Finalmente, para evaluar lo planteado $y$, en especial, para obtener los multiplicadores de producto, ingreso y empleo, tanto en un escenario abierto como cerrado, se compatibiza la información utilizada para el empleo recogidos por el CENSO del año 2002, con la matriz de insumo/producto de la comuna de Punta Arenas utilizada. Para ello se agregó a 29 productos y servicios la matriz ${ }^{4}$, permitiendo realizar el análisis para los subsectores de interés de este estudio: el petróleo y el metanol.

\section{RESULTADOS}

Lo que continua, tiene por ventaja el ayudar a esclarecer el debate entre los reales aportes de cada commodities medido en términos de aporte al desarrollo y sobre la participación histórica que han manifestados los mismo en la economía que se estudia.

Tras revisar los 10 mayores multiplicadores del Tipo I (sistema económico abierto; en anexo tabla 1 y que se resumen en el Fig. 4), se observa que varían desde 1,30 a 2,71, sin embargo, cuando se comparan dichas magnitudes con los del Tipo II (economía cerrada), estos lo hacen desde, 2,27 $(2,03+0,24)$ a $5,71(4,52+1,19)$, siendo ellos servicios de inmobiliarios y de vivienda y, otros minerales respectivamente.

Los valores que se obtiene para los multiplicadores antes descritos, y en especial cuando son comparados con los valores máximos, mínimos y promedios que toman todos ellos, permiten plantear que, cuando se consideran en su totalidad los gastos de los salarios en la economía, la producción se incrementa significativamente. En el caso particular de los commodities, se observa que sólo el petróleo y sus derivados tiene un alto impacto indirecto $(1,17=2,17-1)$ y, para el caso del metanol (industria química), se detecta que su importancia indirecta es baja $(0,98=1,98-1)$, por tanto, no es tan significativo como el del petróleo u otros productos o servicios, en donde se alcanza un multiplicador con un valor máximo de $3.52(3.52=4.52-1)$.

4 Agregación que no tendría repercusiones en las distintas respuestas obtenidas, según el trabajo realizado por Soza- Amigo y Ramos (Revista de Economía Mundial, por aparecer). 
Si lo anterior, se complementa con los multiplicadores del Tipo II (incluye producción inducida más efecto de los salarios; Fig. 4), se observa que, en general, son sustancialmente mayores estos últimos y presentan otro orden de importancia, esto se entiende ocurre en respuesta a las diferencias salariales y uso intensivo de la mano de obra que se da en cada actividad. En este último sentido, nuevamente, destaca el alto impacto total del petróleo $(4,10=3,38+0,63)$ en contraste a la industria química que lo hace con un multiplicador igual a $3,03(2,67+0,36)$, esto confirma que los impactos de la industria química, en general, son menores que los generados por la extracción del petróleo. A lo anterior, se puede agregar que en la extracción del petróleo hay un $25 \%$ más de trabajadores involucrados, por tanto, se entiende y espera que su impacto sea mayor.

En referencia a la importancia global que se desprende de los multiplicadores anteriores, se constata una vez más que son otras las actividades que reportan más vínculos y efectos multiplicadores sobre el resto del sistema productivo, prueba de ello es que los efectos de, por ejemplo, la industria química, son similares a los efectos que se logran con la menor actividad (2.03 versus 2.67 respectivamente) y es menor incluso que el promedio, en tal sentido, solo el petróleo se acerca más al máximo multiplicador y ésta ligeramente por sobre el promedio, sin embargo; aun así, sus efectos son acotados (3,38 versus 4,52 respectivamente).

Lo recientemente expuesto, es interesante pues plantea un debate entre lo que históricamente ha ocurrido en términos de participación en la actividad económica local entre, por un lado, aquellas actividades vinculadas a las producciones de petróleo e industria química (metanol); $\mathrm{y}$, por otro, lo que muestran los distintos multiplicadores en la actualidad.

Recordando que en la primera parte de éste trabajo, se dejó constancia que la producción del petróleo venía sistemáticamente disminuyendo su producción, mientras que en la industria química ella subía, surge la pregunta, ¿conviene más centrar los esfuerzos en una búsqueda por encontrar petróleo; o, basar la economía que se estudia en la producción del metanol (industria química)?, a lo que se tiene que agregar el escenario elegido, en el que se asume que la economía es cerrada, pequeña y aislada geográficamente, en contraste a que si es abierta, a lo que se debe sumar, los efectos reales que se observan en ella por la conmutación, en concreto, se debe revisar, si dadas tales condiciones, se observa la presencia de un efecto de conmutación importante en la economía materia de estudio.

Como una forma de complementar lo expuesto, revísese las actividades con mayores contribuciones al PIB, en tal sentido, se observa una contradicción importante para el caso de la industria química, ello porque su participación en términos de PIB es significativamente muy alto $(34,11 \%)$ en comparación a los otros productos o servicios (como referencia se puede señalar que las 10 actividades que le anteceden tienen en promedio una participación del 4,28\% y, la que se secunda; Administración Pública, un $11,84 \%)$, sin embargo, en sus impactos; o, en la generación de actividades adicionales o complementarias sobre el resto de la economía -lo que a su vez permiten incrementar el desarrollo-, se observa que es muy bajo y en contra de lo que ocurre con las otras actividades.

Por otra parte, en el caso del petróleo, se aprecia una situación más interesante, dado que su aporte al PIB, es en comparación al caso de la industria química de prácticamente 5 veces menor en magnitud (7,14\%), sin embargo, sus aportes en términos de impactos, son mucho mayores, sugiriendo que, aun cuando un commodity presente un alto PIB, no implica que su aporte en términos de desarrollo sea significativo. En este caso pareciera ser que, PIB y actividades directas e indirectas que se consideran son promotoras e inductoras de actividades que acusarían más condiciones en pro de lograr un mayor desarrollo, se encadenan en la medida que menos participación se tenga en el PIB.

Lo anterior, se debe complementar con los beneficios que se logran al incluir en el análisis los efectos inducidos (Anexo tabla 1), esto es, para el caso del petróleo, se tiene un multiplicador de producto igual a 4,01, el cual se descompone como sigue; un multiplicador igual a 2,17 debido a su efecto sobre la producción y 1,84 como efecto inducido $(1,84=$ $4,04-2,17)$, es decir, si aumenta la demanda final en un peso, se incrementaría la producción en forma indirecta en 1,17 pesos, más 1,84 debido al efecto inducido por el incremento del consumo motivado por el gasto de los salarios -asumiendo que los trabajadores gastan sus salarios en la comuna-, con 


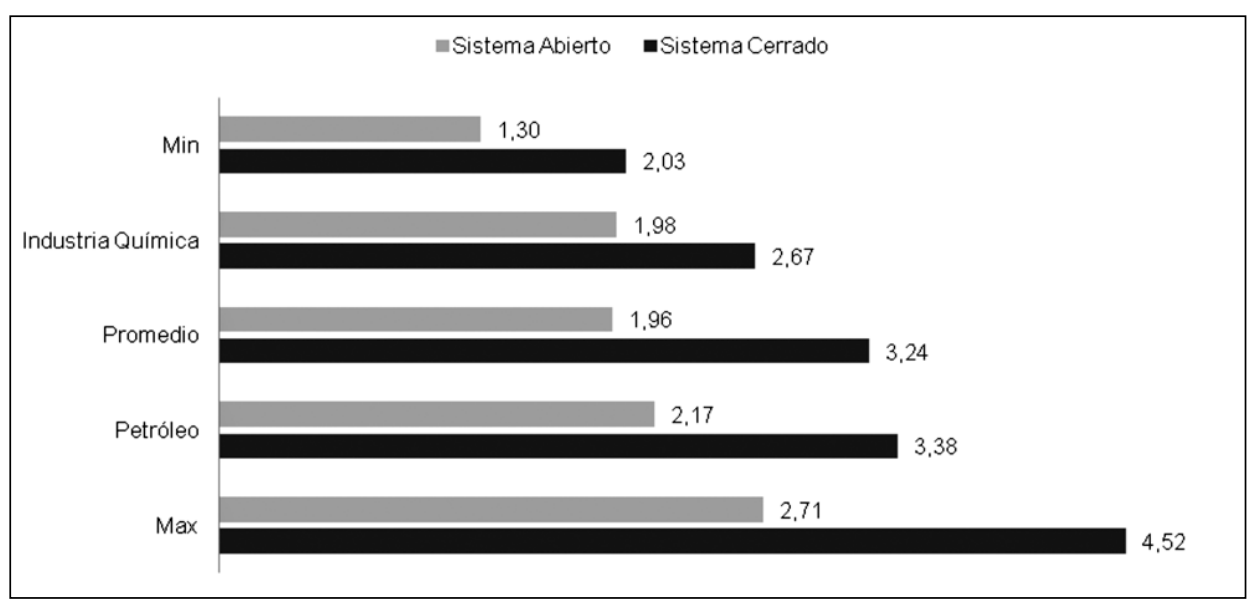

Fig. 4. Multiplicadores de Producto bajo distintos supuestos.

Fuente: Propia en base al anexo 1.

esto la producción en total habría aumentado en 4,01 pesos por el incremento de la demanda final de un peso.

Por otra parte, si se recuerda la composición del PIB y revisa la respectiva tabla input-output que es tomada como referencia en estos análisis, se manifiesta en forma clara que, en éste caso, el alto PIB para la industria química se debe a la magnitud que tienen los excedentes brutos de explotación respecto a los salarios e impuestos, retornos que difícilmente quedan en la comuna.

Otro aspecto que se evalúa, es el concerniente a los efectos particulares de cada commodity -en especial, los referidos a la forma indirecta en que afectan a la producción-, esto es, interesa averiguar cómo se distribuyen los efectos de cada peso gastado, en tal sentido, lo que sigue guarda relación con, el vínculo que se establece entre el incremento de su demanda final con aquellos productos o servicios abastecedores que le permiten al primero incrementar su producción (directa e indirectamente).

Para estudiar lo anterior, se utilizan los multiplicadores de producción del Tipo I y II, para el petróleo y metanol respectivamente (tabla 1 en anexo).

En el caso del petróleo, se observa que, los productos y servicios que más beneficiados se ven cuando se incrementa su producción son, productos químicos $(0,71)$, seguido por el transporte terrestre $(0,12)$, comercio $(0,08)$ y servicios empresariales $(0,06)$, ello indica que, de ocurrir un incremento en la demanda final del petróleo de $\$ 1.000$, la pro- ducción de la industria química lo haría en $\$ 710$, el transporte en $\$ 120$, comercio en $\$ 80$, etcétera.

Por otra parte, si se asume que los salarios se gastan en la comuna, el multiplicador de producción para el petróleo resulta ser de 3,38 (Fig. 4), y el de salarios igual a 0,63 (Fig. 5), lo que entrega un efecto total de 4,01 (en anexo tabla 1, columna $\mathrm{M}$. Producto, sector y salario). En el nuevo escenario, algunos productos o servicios, como, por ejemplo, el transporte se ven altamente favorecidos, en éste caso, su producción casi se duplica, situación similar se observa en el comercio. Un aspecto interesante de ésta parte, es el caso de los productos químicos, en tal condición, su efecto multiplicador en términos relativos pierde importancia -aun cuando el mismo aumenta-, lo que indica que el incremento de los gastos salariales del petróleo en la economía, favorece el desarrollo -vía consumo- de otros sectores.

En el caso de los productos químicos, se observa que el incremento de su producción afecta sustancialmente al petróleo y sus derivados; claro está, que la existencia de gas no aseguran la del petróleo, no así a la inversa, por tal razón, es que su aumento "afecta", entre otros aspectos al segundo.

Marginando lo anterior, se observan dos situaciones interesantes, primero, el efecto multiplicador de la producción cuando se considera a la economía cerrada, el aumenta levemente (de 1,98 a 2,67) en comparación al caso del petróleo (de 2,17 a 3,38), por su parte, el efecto multiplicador de los salarios (Fig. 5), en comparación al resto de actividades, es 


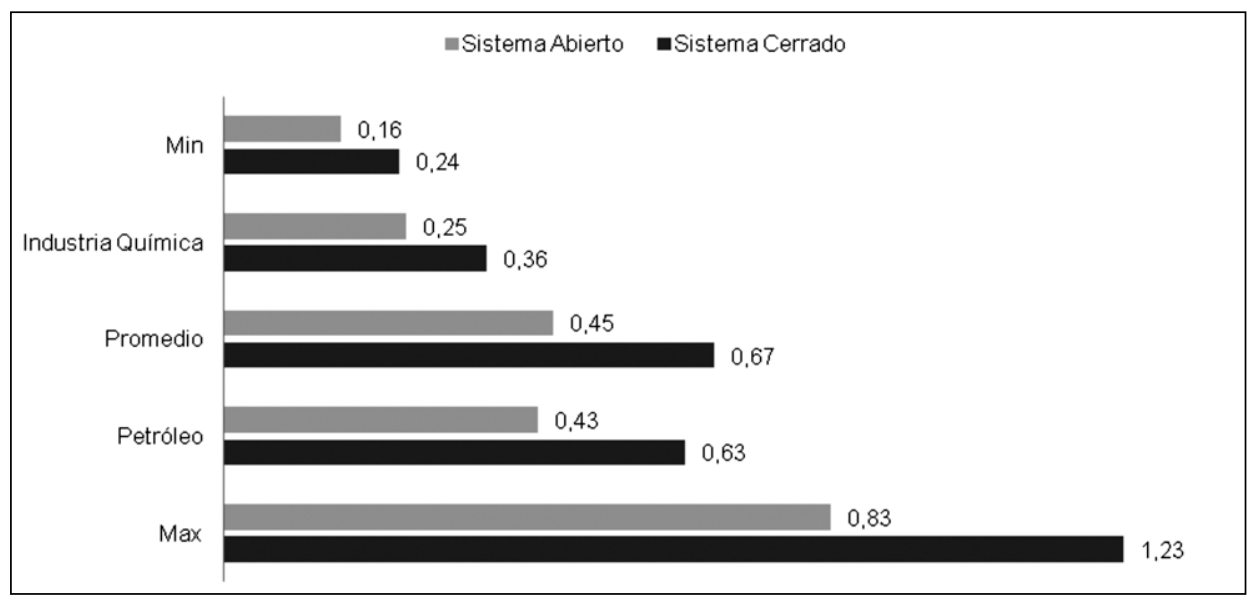

Fig. 5. Multiplicadores de Ingreso asumiendo distintos escenarios.

Fuente: Propia en base al anexo 1.

bajo $(0,36$ lo que prácticamente representa la mitad del obtenido para el petróleo 0,63 ).

Lo expuesto en los párrafos precedentes, confirma que el impacto de los productos químicos, es mínimo en términos globales, e incluso similares a los que menos contribuyen al desarrollo económico local. Además, es inferior al del petróleo, el que por su parte, logra un efecto cercano al promedio y a la mitad de la actividad que logra el mayor efecto multiplicador (Fig. 5).

En segundo lugar, se observa que la magnitud de los multiplicadores de producción para la industria química cuando la economía es cerrada $(2,67$; en anexo tabla 1 y en Fig. 4), son similares a los del petróleo pero, cuando se considera para este último producto una economía abierta $(2,17)$. Es decir, los efectos de los productos químicos, considerando una economía cerrada, son similares a los incrementos que se generan por el petróleo pero, cuando se consideran los multiplicadores para éste del Tipo I, lo cual indica que, el metanol -industria química-, no ayuda -en términos relativos respecto al petróleo $y$ en general- al desarrollo, aun cuando se gasten todos los salarios que se perciben por tal actividad en la economía local.

Otro aspecto interesante de analizar, es el detalle del efecto multiplicador originado por los distintos ingresos (Fig. 5), dado que señala, cuánto reciben los trabajadores por cada peso que se produce en ellos respectivamente, para revisar éste aspecto, se utilizan los multiplicadores de ingreso del Tipo I y II, para el petróleo y la industria química respectivamente.

Para el caso del petróleo, y sobre la base, que el incremento del consumo doméstico es el principal responsable del desarrollo y, no necesariamente el aumento de la producción (Aroca, 2001: 123-124 y, Soza-Amigo y Aroca, 2010: 94), se observa que, cuando la economía ésta abierta, presenta un multiplicador total cercano al promedio $(0,45)$ y algo menos de la mitad que el valor máximo $(0,83)$. Tal valor $(0,43)$ indica que, por cada $\$ 1.000$ pesos que se producen en petróleo, $\$ 430$ aproximadamente se destinan a salarios, los cuales se distribuyen como sigue; $\$ 280$ se pagan en la propia industria, $\$ 70$ en productos químicos y en similar cifra para el resto de actividades. Si tales valores se contrastan con el supuesto que el gasto de los salarios se realiza por completo en la comuna, el multiplicador se ve aumentado en aproximadamente un $70 \%(0,63)$, y en similares proporciones al caso anterior.

En el caso de la industria química, la situación es distinta, y bastante baja en magnitud en comparación al petróleo y sus derivados, incluso sus efectos en términos proporcionales disminuyen para la propia actividad, lo que sería explicado por el leve aumento de producción que se traslada a otros productos o servicios; los que, en general, se ven en algo beneficiados, pero como ya se indicara, en forma muy insipiente, prueba de ello, es que sus magnitudes se acercan a los valores mínimos y son algo menos de la mitad del valor promedio (Fig. 5). 
Otro de los aspectos interesantes de comparar y analizar, es el referido a la generación de empleos por unidad de producto, para ello se recurren a sus respectivos multiplicadores, los cuales indican el número de personas adicionales que se contrata por cada puesto de trabajo que se genera por los distintos productos o servicios (Anexo tabla 1, Fig. 6).

Con respecto al punto anterior, en lo que sigue, se ha optado por modificar el esquema de trabajo, en tal sentido, la forma de abordar ésta parte consiste en analizar sólo el caso del petróleo dada la dispersión que presenta dicha actividad.

Aun cuando se es consientes que, los multiplicadores totales de ambos commodities son semejantes, se da que cuando se considera a la economía abierta, en el caso de los productos químicos, éstos se concentran en un sólo servicio (esparcimiento y otros servicios, 50\%) y, cuando se cierra, éste último baja a un $25 \%$ pero se incrementan en similares proporciones los servicios comerciales e inmobiliarios $y$ de propiedad, por tanto, su efecto es concentrado y muy acotado, a lo que se debe agregar que su valor obedece a el alto excedente bruto de explotación y la baja generación de empleo, lo que distorsiona la realidad e interpretación del mismo.

En relación a los efectos multiplicativos del petróleo en el empleo (Fig. 6), se observa que cuando el sistema es abierto, su efecto en cinco veces mayor que el mínimo y casi el doble que el promedio y, cuando el sistema se considera cerrado sus efectos se triplican y son cercanos a los máximos valores que se obtiene cuando se excluye del análisis la industria química -por las razones ya dadas-, estos antecedentes dan nuevamente muestra de los relevante que es la extracción del petróleo en la economía local, y más importante aún de lo vinculada que ella ésta al resto del sistema productivo.

Respecto caso del petróleo y su efecto multiplicador sobre el empleo, como ya se indicará es desconcentrado; y, cuando la economía se considera abierta varía desde cero (otros productos manufacturados) hasta 1,43 (servicios comerciales) y, cuando está cerrada, desde 0,01 (administración pública) hasta 3,26 (comercio).

En lo referente a los principales efectos multiplicadores del empleo, para el caso del petróleo, se constata que el mayor efecto se logra cuando la economía se considera abierta y se da en el comercio, lo que en la práctica se interpreta como, por cada empleo que se origina en el petróleo se crean 1,5 más en tal servicio. Un aspecto interesante, ocurre cuando se cierra la economía, dado que los efectos multiplicadores cambian en magnitud y orden, tomando con ello relevancia otros productos o servicios que antes eran menos significativos, e.g., los servicios inmobiliarios y propiedad de vivienda o, los servicios de educación. Otro aspecto, llamativo, es la magnitud con que cambian los multiplicadores, en la mayoría de los casos, su valor supera con creces la situación contraria (economía abierta), esto último, da cuenta de la importancia que puede jugar en un análisis posterior el efecto de la conmutación en estos productos.

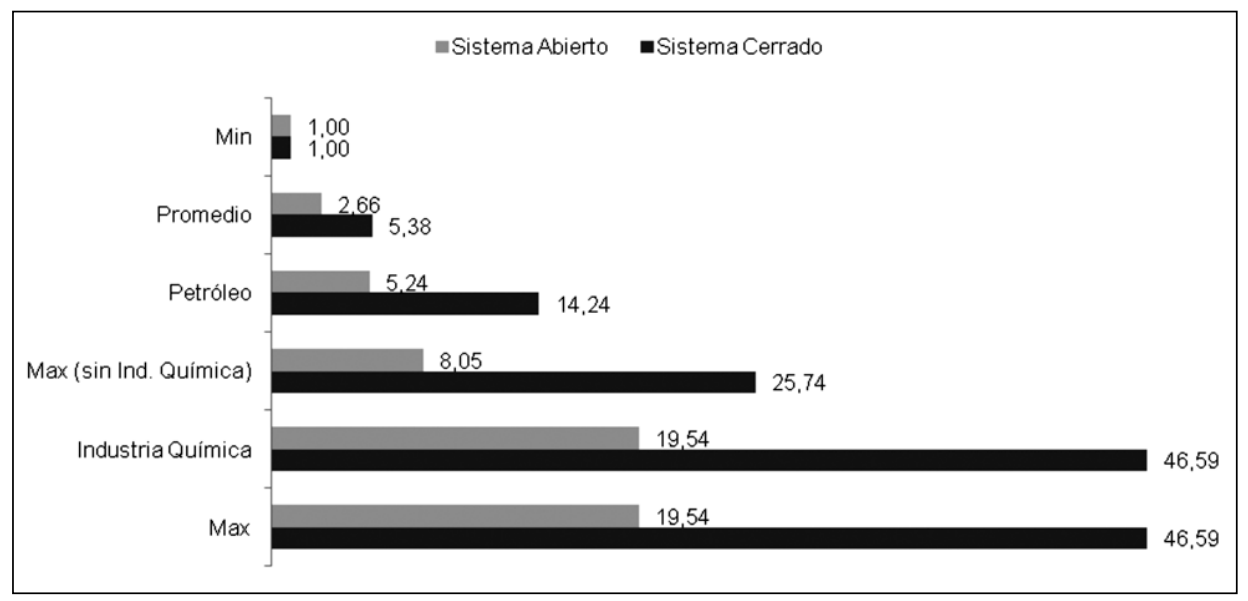

Fig. 6. Multiplicadores de Empleo asumiendo distintos escenarios.

Fuente: Propia en base al anexo 1. 


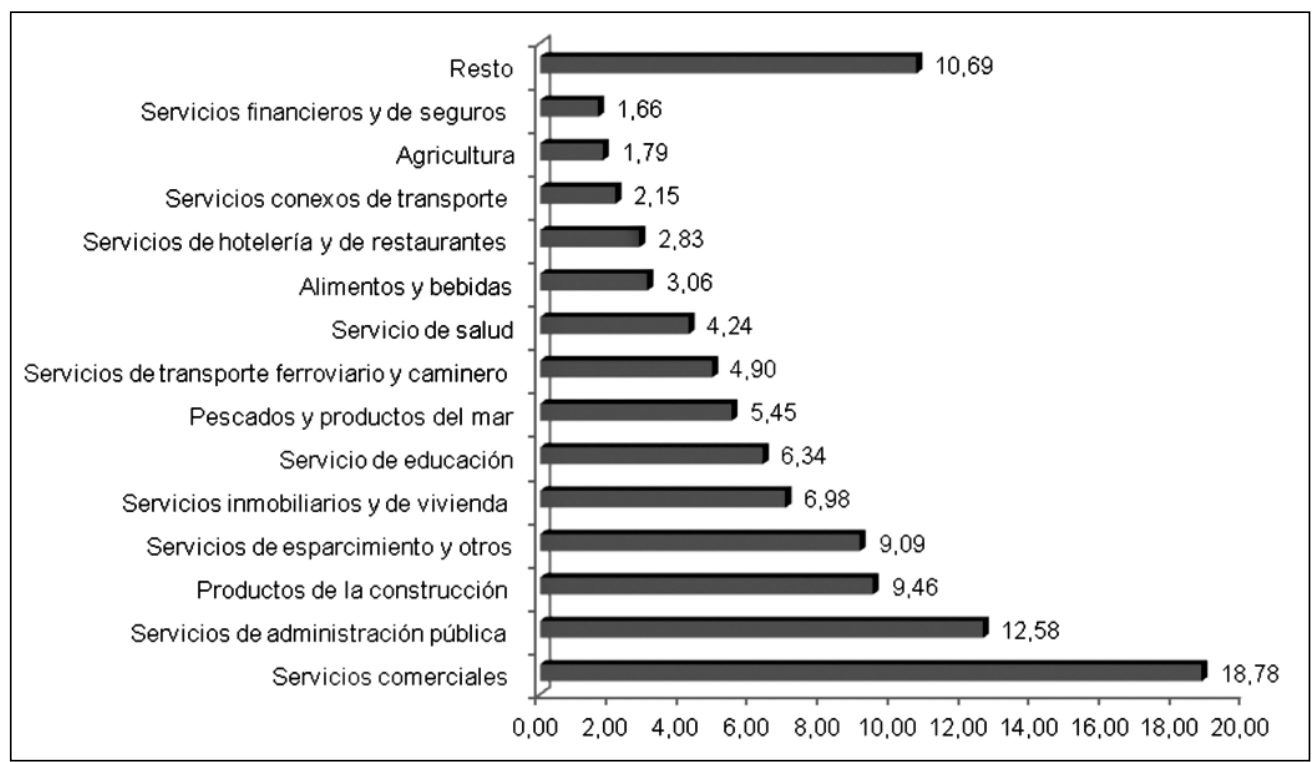

Fig. 7. Principales aportes al empleo en tanto por cíen (Total 40.618 personas). Fuente: Elaboración Propia en base a CENSO del año 2002.

Un aspecto interesante de revisar respecto a lo planteado, es lo concerniente a la pertinencia $y$ validez de la información que se obtiene de los multiplicadores de empleo -en términos de personas por unidad producida por cada actividad-, dado que ellos no nos indican el número de personas que trabajan en cada servicio o producto entregado, en tal sentido, una actividad que presente un alto multiplicador, puede simultáneamente aportar con una baja participación en el conjunto de la economía, para ilustrar este punto, obsérvese la Fig. 7, allí se aprecia que las actividades que más personas requieren, no son precisamente las que tienen un mayor efecto multiplicador en términos de personas por unidad producida.

\section{ENCADENAMIENTOS}

La idea central que existe tras la información que se desprende de los encadenamientos, es la de asociar la demanda y oferta de insumos que se requiere y vende del y hacia el sistema económico por cada producto o servicio transado, por tanto, para el caso que nos ocupa, será un buen indicador dado que manifiesta las dependencias -en términos de movimiento- que genera cada commodities en el resto de la economía, en tal sentido, lo adecuado es considerar un enfoque de demanda y otro de oferta, para así responder a cuáles son los impactos que tienen; por un lado, la demanda y, por otro, la oferta de insumos y productos (intermedios y finales).

En lo que continúa, se revisarán los impactos totales y, directos e indirectos por separado, empleando la propuesta de Rasmussen ${ }^{5} y$, adicionalmente se descompondrá la economía con el objeto de aislar las distintas repercusiones que tiene cada producto o servicio al interior de la economía (planteamiento sugerido por Soza- Amigo en 2007).

El último enfoque tiene por finalidad evaluar -para la actividad que se estudia-, las repercusiones que implican un cambio de su demanda final (df); en, las compras que se demandarán al resto del sistema productivo promovidos por éste shock y, paralelamente, se estudia el efecto que producirá éste último cambio en el resto del sistema económico (para lo cual se excluye su autoconsumo; modelo de demanda); y, en complemento a lo anterior, también se analiza cómo la variación de los inputs primarios (ip) de la actividad estudiada, afecta por

5 En estricto rigor, Rasmussen se basa sólo en el modelo de Leontief para determinar ambos índices, sin embargo, fue a fines de los años 60 y principios de los 70, con los trabajos de Ghosh, A. (1968) y Augustinovics, M. (1970), en donde se inicia un fuerte debate sobre el uso conjunto de los modelos de Demanda (Leontief) y oferta (Ghosh), aspecto que son considerados y aceptados en éste trabajo. 
medio de sus ventas al resto del sistema productivo de la economía, tanto en forma directa como indirecta (excluido su autoconsumo; modelo de oferta), esto es, se analiza cómo la variación de los ip de la actividad estudiada, afecta las compras que el resto del sistema productivo realiza sobre ésta y simultáneamente, se observa cómo estas últimas afectan tanto directa como indirectamente a lo que queda del sistema económico, aislando su autoconsumo y las repercusiones que tiene tal variación sobre su propia producción.

Basados en la propuesta de Rasmussen (tabla 1 de anexo), se observa que en el caso del petróleo, correspondería a una actividad del tipo impulsora de la economía cuando se consideran todos los efectos (directos e indirectos) ${ }^{6}$, situación similar se observa cuando se analizan los efectos indirectos totales $\left(\mathrm{BL}^{(\mathrm{Z}-\mathrm{I})}\right.$ y $\mathrm{FL}^{(\mathrm{B}-\mathrm{I})}$, respectivamente), dado que aumenta su encadenamiento hacia atrás (en un $14 \%$ ) pero disminuye el que va hacia adelante (en un $8 \%$ ). Sin embargo, cuando se consideran sólo los efectos indirectos hacia otras industrias $\left(\mathrm{BL}^{(\mathrm{z}-\mathrm{z})}\right.$ y $\left.\mathrm{FL}^{(\mathrm{B}-\mathrm{b})}\right)$, queda de manifiesto que tal actividad es clave, es decir, presenta respecto al promedio una alta demanda y oferta de insumos y; además, tiene una alta dispersión (poca concentración) de sus efectos a lo largo de todo el entramado económico, esto es, desde la perspectiva de la demanda y oferta de insumos $\left(\mathrm{v}_{\mathrm{j}} \mathrm{y}_{\mathrm{i}}\right.$, respectivamente).

En el caso de la industria química, se observa una situación similar, aunque contraria a la anterior. Similar dada la magnitud de los encadenamientos y, contraría, pues más bien responde a una actividad del tipo base y no impulsora cómo la anterior?. Por otra parte, en términos relativos, se observa que los efectos indirectos que genera son muy inferiores a los obtenidos para el caso del petróleo, y en el caso de sus efectos indirectos sobre otras industrias, estos son más bien base, con un alto acento en la oferta de insumos que hace (tabla 1 en anexo).

Finalmente, cuando las actividades anteriores se analizan bajo el esquema sugerido por SozaAmigo (2007), se observa que ellas responden a una tipología de clasificación que es del tipo "clave",

6 Aquella que demanda insumos por sobre el promedio, pero ofrece menos en comparación al resto.

7 Actividad base es aquella que ofrece insumos o productos finales por sobre el promedio, pero demanda poco en comparación al resto. alta demanda y oferta de insumos en los términos que estos indicadores miden $\left(\mathrm{i}^{\mathrm{t}} \mathrm{G}_{22} \mathrm{~A}_{21} \mathrm{y}_{1}\right.$; para la el encadenamiento hacia atrás y $r_{1} B_{12} W_{22} i_{t}$ para el encadenamiento hacia adelante), tal definición es reforzada y entendible porque además ambas actividades presentan una alta dispersión ponderada (tabla 1 en anexo).

Aun cuando lo anterior, es interesante dado que da una perspectiva de lo influyente que es cada commodity en la economía materia de estudio, llama la atención que éstos commodities, presentan efectos indirectos bajos en general cuando son comparados con la totalidad de actividades de la economía, independientemente del tipo de tipología que se trate (impulsor, base o isla), lo interesante de este último aspecto, es el contra sentido que se observa con indicadores tales como el PIB.

Considerando que la mayor participación en el PIB era el de la industria química con un 34,11\% $y$, en menor grado el petróleo (cuarto lugar con una participación del 7,14\%), se observa que luego de realizar un análisis más holístico que el petróleo en la práctica influye más en la economía que la industria química, pero en ningún caso ambos logran efectos superiores al promedio.

\section{LA CONMUTACIÓN EN LA COMUNA DE PUNTA ARENAS}

Otro de los aspectos que se ha creído oportuno revisar es, el referido a la conmutación y su relación con los commodities aquí analizados, ello dado que la misma se asocia a lugares de trabajo, residencia y gasto de salarios en localidades distintas a las elegidas para laborar, por tanto, se relaciona con el sistema de trabajo impuesto, esto es, sistema económico abierto y cerrado.

La conmutación en éste caso, debe ser entendida como la relación que existe entre, por un lado, las personas que trabajan y por tanto, reciben sus salarios en la comuna de Punta Arenas; y, por otro, con la residencia de estas últimas, la que se entiende es en otras localidades.

Lo planteado permite asumir que los gastos que los conmutantes efectúan con lo que se asume son ingresos percibidos por los servicios prestados en la comuna de Punta Arenas, generan una suerte de pérdidas de demandas para ésta última, lo que perjudica a la comuna pero, beneficia a las que los 
trabajadores han elegido para vivir con el aumento de producción generado con los recursos que se perciben, en este caso, en Punta Arenas.

La Fig. 8, muestra los lugares de residencia de los conmutantes de la comuna de Punta Arenas, allí se observa que en total son 496 las personas que trabajan en Punta Arenas, en este caso, sus residencias y cantidad son señaladas con las flechas de destino desde la citada comuna hacia el resto del país.

De los conmutantes queda claro que la gran mayoría tiene como lugar de residencia la zona central del país (aproximadamente el 50\%), casi el doble de los que conmutan en la propia región (alrededor del 25\%).

Por otra parte, se observa del CENSO del año 2002, que el mayor número de conmutantes por actividad económica, se dan en el petróleo siendo ésta del orden del 3,77\% y, en los productos químicos sólo alcanzaría al 2,11\%, es decir, la conmutación en estos commodities, es más bien baja, sin embargo, $y$ dado que sus salarios son altos, sus efectos son significativos, a modo ilustrativo, la renta media según el Instituto Nacional de Estadísticas por actividad es

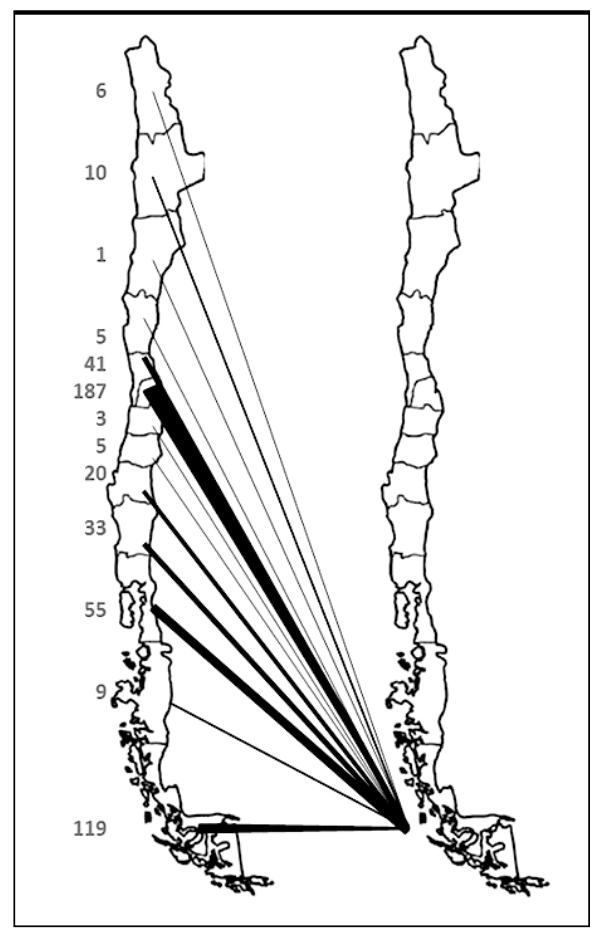

Fig. 8. Conmutantes a la Comuna de Punta Arenas. Fuente: Elaboración Propia en base a CENSO del año 2002. para el caso de la minería 2,75 veces mayor que el promedio para el año 2003, luego queda claro que los efectos de la conmutación son significativos en estos commodities.

\section{DISCUSIÓN}

Uno de los puntos que, es natural genere dudas sobre los resultados obtenidos, tienen que ver con la magnitud de algunos multiplicadores; en especial, los del empleo en la industria química, al respecto hay algunos antecedentes a considerar y que justifican tales valores.

Por un lado, se da que, en aquella industria en particular $y$, en concreto en la dedicada a la elaboración del metanol, existe una alta contratación se servicios, casinos (alimentación), seguridad, correos, etc., dejando en la práctica, una relación alta entre el número de Directores, Profesional y Técnicos versus los que se desempeñan en el Servicio Administrativo, ello en parte justifica los altos salarios respecto a otras actividades, a lo que se debe sumar, los distintos incentivos que se dan en aquella industria, y que a su vez también explica lo bajo del total de contratados.

Por otra parte, existe un hecho no menor, $y$ que efectivamente afecta a los resultados, se debe recordar que se trabaja con dos fuentes te datos, las que si bien es cierto tienen una data muy próxima, no es la misma (CENSO-2002 y una Tabla InsumoProducto para la comuna del año 2003).

Lo anterior trae consecuencias sobre los contratados, salarios y niveles de producción; $y$, en especial, en los de la industria química producto del cambio que se da en la producción del año 2003 respecto al 2002. A modo de información ilustrativa, considere la Encuesta Nacional Industrial Anual para el año 2007 (ENIA-2007), en ella se aprecia que en la región se da un fuerte shock en la producción y contratación de mano de obra en esa actividad y, ocurre precisamente entre los años 2002 y 2003 (Fig. 9). Nótese que para el año 2002 laboraron en el sector químico 232 personas, a eso agregue que la misma fuente señala que para el año 2003 en dicha actividad trabajaron 1527, es decir, del año 2002 al 2003 se incrementó el número de contratados directos en un $658.20 \%$, ahora considere -al igual como se hace en el estudio realizado-, que se mantiene los trabajadores del año 2002 en el 


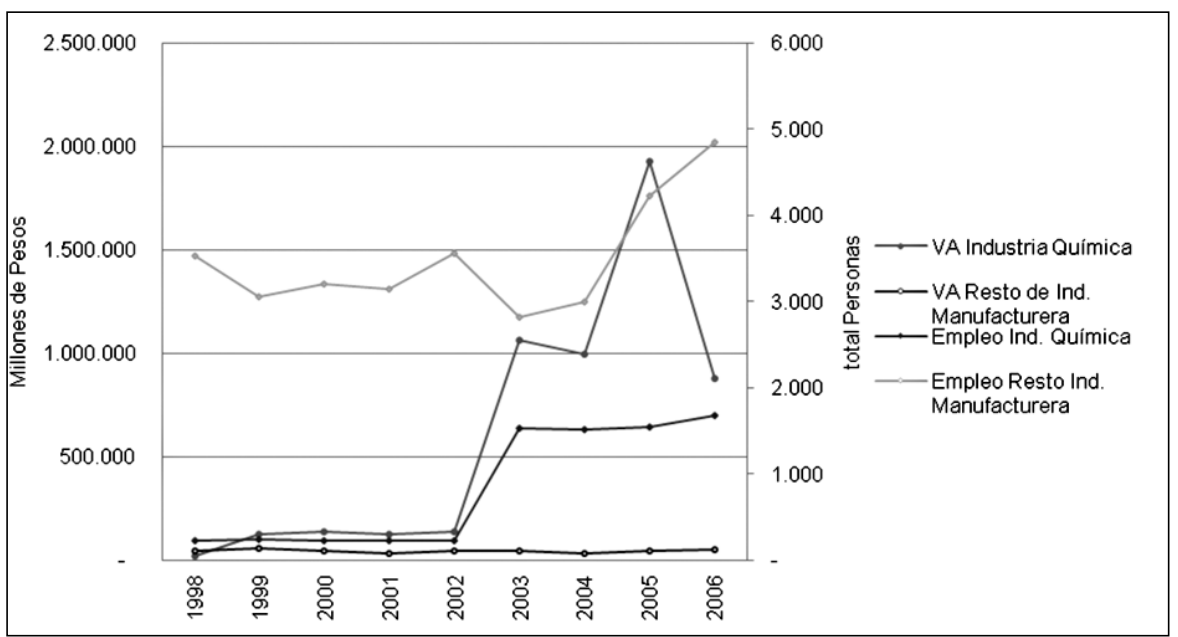

Fig. 9. Valor Agregado (millones de pesos) y Contratados en Industria Manufacturera para Magallanes (1998- 2006). Fuente: Propia en base a ENIA- 2007.

2003, lo que es equivalente, a asumir el cruce del CENSO-2002 y la matriz del 2003, la consecuencia es clara, se tendrá que la renta promedio de los trabajadores del año 2003, utilizando los contratados en 2002, es mucho mayor y distinta a la 2003, eso evidentemente distorsiona los resultados.

Una alternativa para evaluar la distorsión en los resultados obtenidos en la industria química, es repetir el ejercicio pero empleando datos de la ENIA-2007 y, paralelamente asumir que el resto de las actividades mantuvieron su estructura laboral (número de empleados y renta) para los años 2002 y 2003.
Del ejercicio propuesto en el párrafo antepuesto, se observa un panorama distinto en cuanto a magnitud de impactos, pero igualmente concluyente en la forma en que se afecta, es decir, se reducen los impactos, pero se mantiene, en general, el orden de los efectos (Fig. 10).

Analizando los multiplicadores de empleo bajo el nuevo supuesto, se observa que en el caso del petróleo su multiplicador nuevamente es algo más de cinco veces mayor que el menor, el doble del promedio y levemente superior al máximo valor cuando el sistema es abierto.

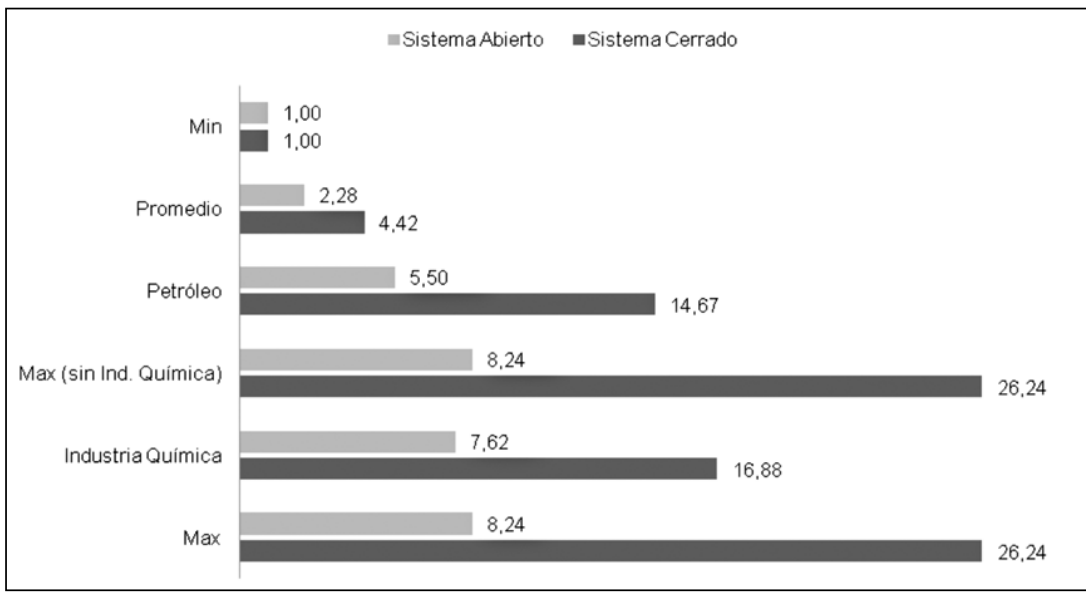

Fig. 10. Multiplicadores de Empleo asumiendo 1537 empleos en Industria Química y distintos sistemas. Fuente: Propia en base al anexo 1 tabla 2. 


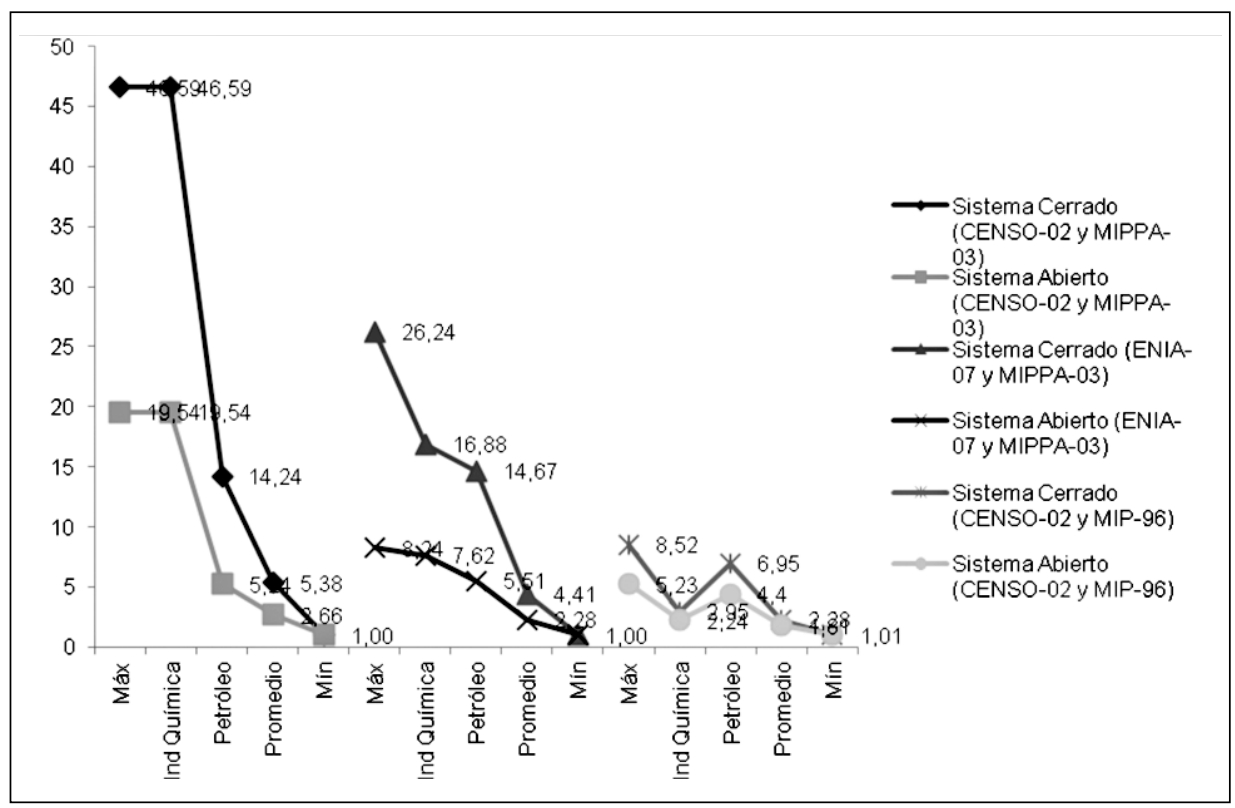

Fig. 11. Multiplicadores de Empleo utilizando distintas matrices sistemas. Fuente: Propia en base al anexo 1 tabla 2.

Cuando se asume un sistema cerrado, se observa que el mismo aumenta ligeramente respecto a la situación original (trabajadores del CENSO-2002), y mantiene su representatividad respecto al supuesto anterior, por tanto no se ven grandes cambios.

En el caso de la industria química y su multiplicador de empleo, en el nuevo supuesto y, como era de esperar su impacto se ha visto significativamente disminuido. En el sistema abierto disminuye prácticamente en tres veces y, en algo más del doble en el cerrado, lo que confirma que los efectos reales de tal actividad sobre la economía que se estudia, no son tan importante como se pudiera esperar, en tal sentido, se confirma que sus impactos son similares a los del petróleo y menores que los de otras actividades.

Otra forma -aunque aproximada- de comprobar lo discutido, es utilizando la matriz regional del año 1996 y los datos del CENSO del año 2002, de tal ejercicio se observa nuevamente el mismo comportamiento en términos de impacto y orden que en las otras situaciones, con la pequeña excepción de un cambio en el impacto para el petróleo, el cual obedecería a que ahora sus efectos son considerados a nivel regional, mientras que la industria química ello no ocurre, dado que sólo se procesa metanol en la comuna de Punta Arenas (anexo tabla 2 y Fig. 11 resumen lo comentado).

Finalmente, se puede indicar que aún cuando se han considerado distintos escenarios y años de evaluación, se observa que los impactos, del petroleo e industria química, en general, son bajos, no aportando al desarrollo local como otras actividades.

\section{CONCLUSIONES}

Para finalizar, se pasan a presentar las principales conclusiones del trabajo realizado, las que se enuncian siguiendo el orden en que fueron tratados los distintos enfoques de ésta investigación.

En referencia a los efectos multiplicativos que tienen los commodities, se observa que ellos, en general, son dispares, mientras el petróleo presenta altos y dispersos efectos multiplicativos, ya sea en términos de producción, ingreso o empleo, considerando a la economía abierta y cerrada, se cae en el contraste que ellos son bajos y concentrados en la industria química (metanol).

Por otra parte, cuando se analiza la participación que tienen los commodities en la economía en términos de PIB, se observa que para la industria química es muy alto y concentrado (primer lugar, 
con un tercio del total), y para el petróleo, se sitúa en el cuarto lugar, con un escaso 7,14\%.

Por otra parte, en el caso de los commodities se observa que, un alto porcentaje de participación en el PIB, no implica que su aporte en términos de desarrollo sea significativo, en este sentido, pareciera ser que, PIB y actividades que son promotores o inductoras de dinamismo económico, se vinculan, en la medida que menos participación se tenga en el PIB, lo cual se puede esbozar como sigue "los commodities -al menos los aquí estudiados- al ser productos que sólo se exportan, generan poca demanda y oferta de insumos hacia el resto del sistema económico local, no ayudando por tanto en forma significativa al desarrollo del mismo". La justificación de lo planteado, se debería a que el alto PIB que manifiestan los commodities, se debe a la magnitud que tienen los excedentes brutos de explotación respecto a los salarios e impuestos, beneficios que difícilmente quedan en la comuna.

Desde otra perspectiva, se observa que la magnitud de los multiplicadores de producción para la industria química cuando la economía es cerrada $(2,67)$, son similares a los del petróleo pero, cuando se considera para éste último producto una economía abierta $(2,17)$. Es decir, si se comparan los efectos que traería el gastar todos los pagos recibidos por los factores productivos de la industria química en la totalidad en la economía versus los que se generan por el petróleo, pero asumiendo que ellos no se gastan en forma local, aun así, los primeros, no alcanzan a cubrir las consecuencias que se manifiestan por explotar el petróleo, esto es, las repercusiones que traen los productos químicos, considerando una economía cerrada, son similares a los incrementos que se generan por el petróleo pero, cuando se consideran los multiplicadores para éste del Tipo I, lo cual indica que, el metanol -industria química-, no ayuda -en términos relativos- al desarrollo, aun cuando se gasten todos los salarios que se perciben por tal actividad en la economía local, en ésta línea, se observa que existen otros productos y servicios que aportan más al desarrollo local, por ejemplo, la agricultura y construcción.

Por otra parte, haciendo un símil entre las distintas consecuencias que trae el multiplicador de ingreso y considerando ambos escenarios (economía abierta y cerrada), llamó la atención que, en el caso de los productos químicos, su efecto multiplicador, aun cuando aumenta, ocurre que, en términos relativos pierde importancia. En tal sentido, sus implicancias son menores que en el caso del petróleo, lo que indica que el aumento de los gastos salariales del petróleo en la economía, favorecen más el desarrollo en comparación a la industria química, dado que el primero incrementa el consumo de otros productos o servicios.

Además de lo anterior, también se observan que; por una parte, se manifiesta lo importante que son ambas actividades -en términos de PIB- en los tipo de sectores a los que pertenecen (primarios e industria respectivamente, en ambos casos son las más importantes); y, en segundo lugar, que en términos generales, se aprecia que no son actividades relevantes en un sentido de los efectos que generan en la globalidad de la economía, en tal línea, tras realizar un análisis generalizado, se observa que actividades más ligados a los servicios, presentarían una alta participación en el PIB y además altos efectos sobre la economía, esto es, ellos presentarían unos efectos totales e indirectos más significativos que el de los commodities aquí estudiados, a lo que se debe agregar, sus mayores efectos multiplicativos de producto (economía abierta), de salarios e ingresos.

Lo expuesto en los párrafos precedentes, confirma que el impacto de los productos químicos, es mínimo en términos globales e inferiores a los del petróleo.

En lo referente a la conmutación, se detectó que en estos commodities, es más bien baja, sin embargo, y dado que los salarios asociados a tales productos son altos, sus efectos son significativos.

En referencia a las hipótesis establecidas, y a modo de resumen se puede plantear que, se observa que efectivamente los commodities presentan una participación importante en el PIB, pero no aportan al desarrollo local, en términos de, altos impactos directos o indirectos o, en términos de, multiplicadores de empleo o ingreso. Con respecto a la segunda hipótesis, efectivamente se comprueba del estudio realizado una falta de correlación importante entre encadenamientos y multiplicadores de ingreso y empleo, ello se debería a que en los últimos - y en los encadenamientos bajo un contexto clásico- no se consideran los impactos de los inputs primarios.

Para finalizar, se presentan algunas Políticas que se pueden implementar en pro de lograr un desarrollo mayor por medio del impacto de los 
commodities, en primer lugar, se detecta que al existir una alta exportación de ellos, se produce una suerte de pérdida de desarrollo, por tanto, se deben buscar actividades que permitan gestar otras industrias basadas en estos commodities, en ésta línea, se emplearía como insumo el uso de estos; en especial, se sugiere hacer uso del metanol, con el fin de inducir otras actividades económicas.

Por otra parte, se considera interesante, el legislar sobre el cobro de un mayor impuesto o royalty por la explotación de este tipo de productos, a lo que se debe sumar que su recaudación quede a nivel local y no se pierda en un gasto que este referido a nivel nacional.

A lo anterior, se debe agregar que se detecta que existe una fuga importante de utilidades, en el sentido, que los dueños del capital no gastan sus beneficios en la comuna, por tanto, se sugiere que una parte de las utilidades se trasformen en otros proyectos de inversión; o bien, en ayudas sociales "importantes".

Generar, vínculos entre el lugar de trabajo y la residencia de las personas, esto es, sentar las bases para que la comuna sea atractiva en términos de residencia para aquellos que conmutan en esta.

\section{BIBLIOGRAFÍA}

AROCA, P. 2004. Migración Interregional en Chile Modelos y Resultados 1987-2002. Revista Notas de Población 78:97-154.

2001. Impacts and development in local economies based on mining: The case of the Chilean II region. Resources Policy, (27):119-134.

AROCA, P. y M. ATIENZA 2011. Economic implications of long distance commuting in the Chilean mining industry. Resources Policy, doi:10.1016/j.resourpol.2011.03.004. 2008. La Conmutación Regional en Chile y su Impacto en la Región de Antofagasta. Revista EURE, XXXIV(102): 97-120.

AROCA, P. y G. HEWINGS 2002. Migration and Regional Labor Market Adjustment: Chile 1977-1982 and 1987-1992.

The Annals of Regional Science, 36:197-218

AROCA, P. y W. MALONEY 2005. Migration, Trade, and Foreign Direct Investment in Mexico. World Bank Economic Review, Oxford University Press, 19(3):449-472.

AROCA, P., G. HEWINGS y J. PAREDES 2001. Migración Interregional y el Mercado Laboral. Cuadernos de Economía, 27(2): 321-345.
AUGUSTINOVICS, M. 1970. Methods of International and Intertemporal Comparison of Structure. In: Contributions to input-output analysis. Carter Anne y Andrew Bródy, eds. Amsterdam, New York, Oxford, North-Holland Publishing Company, pp. 249-269.

BANCO CENTRAL DE CHILE 2006. Cuentas Nacionales: 1996- 2005. Banco Central de Chile, Santiago. 2007. Cuentas Nacionales: 2003- 2006. Banco Central de Chile, Santiago.

2008. Cuentas Nacionales: 2003- 2007. Banco Central de Chile, Santiago.

GHOSH, A. A Note on Leontief Models with Non-Homogeneous Production Functions. En: Planning programming and Input-output models: Selected papers on Indian planning. Monographs, University of Cambridge Department of Applied Economics at the University press, New York, 1968. p 45.

HEWINGS, G. 1985. Regional Input-Output Analysis. Sage Scientific Geography Series 7, Beverley Hills.

HEWINGS, G., M. FONSECA y M. SONIS 1989. Key Sectors and Structural Change in the Brazilian Economy: A comparison of alternative approaches and their policy implications. Journal of Policy Modeling, 11(1):67-90.

HIRSCHMAN, A. 1958. The Strategy of Economic Development. Yale University Press. New Haven, Connecticut.

ISARD, W. y R. KUENNE 1953. The Impact of Steel Upon the Greather New York-Philadelphia Industrial Region. The Review of Economics and Statistics, 35(4):289-301.

ISARD, W., I. AZIS, M. DRENNAN, S. SALTZMAN y E. THORBECKE 1998. Methods of Interregional and Regional Analysis, Ashgate, Hants, UK.

KEYNES, J.M. 1936. The General Theory of Employment, Interest and Money. Macmillan Cambridge University Press, for Royal Economic Society, Cambridge.

KRUGMAN, P. y M. Obstfeld 2006. Economía Internacional. Pearson Educación, Madrid.

LEONTIEF, W. 1941. The Structure of American Economy, 1919-1939. Oxford University Press, New York.

MIDEPLAN 1998. Evolución de la actividad económica regional 1985-1996: análisis del Producto Interno Bruto desde un enfoque nacional/regional. Ministerio de Planificación y Cooperación. Andros Impresores, Santiago. 2005. Aproximación a las Economías Regionales con base en las Matrices de Insumo Producto Regionales del Año 1996. Ministerio de Planificación, Santiago. pp. 211.

MILLER, R. 1957. The Impact of the Aluminum Industry on the Pacific Northwest: A regional input- output analysis. The Review of Economics and Statistic, 39(2):200-209. 
MILLER, R. y P. BLAIR 2009. Input-Output Analysis: Foundations and Extensions. Cambridge University Press.

MIYAZAWA, K. 1976. Input-Output Analysis and the Structure of Income Distribution. Springer-Verlag, New York.

PAREDES, D. y P. AROCA 2008. Metodologías Para Estimar un Índice Regional de Costo de Vivienda en Chile. Cuadernos de Economía, 46(134):129-143.

PULIDO, A. y E. FONTELA 1993. Análisis Input-Output. Modelos Datos y Aplicaciones. Editorial Pirámide, Madrid.

RASMUSSEN, P. 1956. Studies in Inter-Sectoral Relations. North- Holland P. C., Amterdam.

SOZA-AMIGO, S. y C. RAMOS 2011. La Agregación en las Tablas Input-Output: Una revisión desde la perspectiva de las ramas que no se unen. Revista de Economía Mundial, en prensa.
SOZA-AMIGO, S. y P. AROCA 2010. Oportunidades Pérdidas en Magallanes. Magallania, 38(2):89-101.

SOZA-AMIGO, S. 2007. Análisis Estructural Input-Output: Antiguos problemas y nuevas soluciones. Tesis Doctorado en Economía Aplicada. Oviedo, España, Universidad de Oviedo, Departamento de Economía Aplicada.

2008a. Análisis Comparativo para la Economía Magallánica desde la Perspectiva del Enfoque Input-Output. Revista de Análisis Económico, 23(2):79-102.

2008b. Interrelaciones Sectoriales en Magallanes: En busca de su estructura económica. Magallania, 36(1):79102.

2010. La Identidad Magallánica Dados Los Principales Cambios Económicos y Estructurales Ocurridos en Chile Entre 1960 y 2008. Magallania, 38(1):109-136. 


\section{ANEXOS}

Formulaciones que determinan el tipo de encadenamiento según las distintas técnicas empleadas (hacia atrás $(\mathbf{B L})$ y delante $(\mathbf{F L}))^{8}$.

Ventas interindustriales $\left(\mathbf{z}_{\mathbf{i j}}\right)+$ demanda final $\left(\mathbf{c}_{\mathbf{i}}\right.$; consumo, ofd; resto de demanda final) $=$ ventas totales $\left(\mathbf{x}_{\mathbf{i}}\right)$ $\sum_{j=1}^{n} z_{i j}+c_{i}+o f d_{i}=x_{i}$

Compras interindustriales $\left(\mathbf{z}_{\mathbf{i j}}\right)+$ valor agregado $\left(\mathbf{w}_{\mathbf{j}}\right.$ : salarios; $\mathbf{o v}_{\mathbf{j}}$ : resto del valor agregado)+ importaciones $\left(\mathbf{m}_{\mathbf{j}}\right)=$ salidas totales $\left(\mathbf{x}_{\mathbf{j}}\right)$

$\sum_{\mathrm{j}=1}^{\mathrm{n}} \mathrm{z}_{\mathrm{ij}}+\mathbf{w}_{\mathrm{j}}+0 \mathrm{v}_{\mathrm{j}}+\mathrm{m}_{\mathrm{j}}=\mathbf{x}_{\mathrm{j}}$

Matriz de Coeficientes Técnicos

$A=a_{i j}=\frac{Z_{i j}}{x_{j}}=\begin{array}{cccc}a_{11} & a_{12} & \cdots & a_{1 n} \\ \vdots & \vdots & \ddots & \vdots \\ a_{n 1} & a_{n 2} & \cdots & a_{n n}\end{array}$

Matriz Inversa de Leontief

$(I-A)^{-1}=\left[b_{1 j}\right]=\begin{array}{cccc}b_{11} & b_{12} & \cdots & b_{1 n} \\ \vdots & \vdots & \ddots & \vdots \\ b_{n 1} & b_{n 2} & \cdots & b_{n n}\end{array}$

Multiplicador de Producción

$\mathbf{O}_{\mathrm{j}}=\sum_{-1}^{\mathrm{n}} \mathbf{b}_{\mathrm{ij}}$

Rasmmusen (1956)

Poder de Dispersión

$B L_{j}{ }^{R}=\frac{n i^{t}(I-A)^{-1}}{i^{t}(I-A)^{-1}}$

Efectos Indirectos (Leontief)

$\mathbf{B L}^{(\mathbf{Z}-\mathbf{I})}=(\mathbf{I}-\mathbf{A})^{-1}-\mathbf{I}$

E. Indirectos sobre Industrias (Leontief)

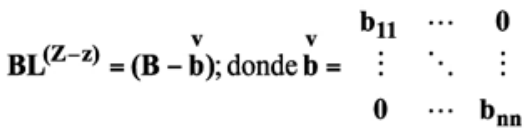

Soza- Amigo (2007; descomposición)

$$
\begin{aligned}
& \mathbf{P B L}_{\mathrm{j}}^{\mathrm{c}, \mathrm{p}}=\left(\mathrm{i}^{\mathrm{t}} \mathbf{G}_{22} \mathbf{A}_{21} \mathbf{y}_{1}\right) \mathbf{p}_{\mathrm{j}}
\end{aligned}
$$

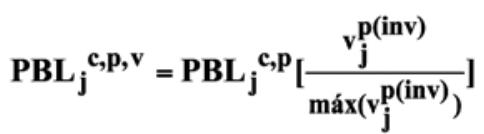

Multiplicador de Ingreso

$$
\mathbf{H}_{\mathrm{j}}=\sum_{\mathrm{i}}^{\mathrm{n}} \frac{\mathbf{w}_{\mathrm{i}}}{\mathbf{x}_{\mathrm{i}}} \mathbf{b}_{\mathrm{ij}}
$$

Matriz de Coeficientes de Distribución

$$
\overrightarrow{\mathbf{A}}=\overrightarrow{\mathbf{a}}_{\mathrm{ij}}=\frac{\mathbf{Z}_{\mathrm{ij}}}{\mathbf{x}_{\mathrm{i}}}=\begin{array}{cccc}
\overrightarrow{\mathbf{a}}_{11} & \overrightarrow{\mathbf{a}}_{12} & \cdots & \overrightarrow{\mathbf{a}}_{1 \mathrm{n}} \\
\vdots & \vdots & \ddots & \vdots \\
\overrightarrow{\mathbf{a}}_{\mathrm{n} 1} & \overrightarrow{\mathbf{a}}_{\mathrm{n} 2} & \cdots & \overrightarrow{\mathbf{a}}_{\mathrm{nn}}
\end{array}
$$

Matriz Inversa de Ghosh

$$
\overrightarrow{\mathbf{B}}=(\mathbf{I}-\overrightarrow{\mathbf{A}})^{-1}=\left[\overrightarrow{\mathbf{b}}_{1 \mathrm{j}}\right]=\begin{array}{cccc}
\overrightarrow{\mathbf{b}}_{11} & \overrightarrow{\mathbf{b}}_{12} & \cdots & \overrightarrow{\mathbf{b}}_{1 \mathrm{n}} \\
\vdots & \vdots & \ddots & \vdots \\
\overrightarrow{\mathbf{b}}_{\mathrm{n} 1} & \overrightarrow{\mathbf{b}}_{\mathrm{n} 2} & \cdots & \overrightarrow{\mathbf{b}}_{\mathrm{nn}}
\end{array}
$$

Multiplicador de Empleo

$$
\mathbf{E}_{\mathrm{j}}=\sum_{\mathrm{i}=1}^{\mathrm{n}} \frac{\left(\mathrm{(Li}_{\mathrm{i}} / \mathrm{x}_{\mathrm{i}}\right)}{\left(\mathrm{L}_{\mathrm{j}} / \mathrm{x}_{\mathrm{j}}\right)} \mathbf{b}_{\mathrm{ij}}
$$

Sensibilidad de Dispersión

$F_{i}{ }_{i}^{R-G}=\frac{n(I-B)^{-1} i^{t}}{i(I-B)^{-1} i^{t}}$

Efectos Indirectos (Ghosh)

$\mathbf{F L}^{(\mathbf{B}-\mathbf{I})}=\overrightarrow{\mathbf{B}}-\mathbf{I}=(\mathbf{I}-\overrightarrow{\mathbf{A}})^{-1}-\mathbf{I}$

E. Indirectos sobre industrias (Ghosh)

$\mathbf{F L}^{(\mathbf{B}-\mathbf{b})}=\left(\overrightarrow{\mathbf{B}}-\overrightarrow{\mathbf{b}}^{\prime \mathbf{v}}\right) ;$ donde $^{\overrightarrow{\mathbf{b}}^{\prime} \mathbf{v}}=\begin{array}{ccc}\overrightarrow{\mathbf{b}}_{11} & \cdots & \mathbf{0} \\ \vdots & \ddots & \vdots \\ \mathbf{0} & \cdots & \overrightarrow{\mathbf{b}}_{\mathrm{nn}}\end{array}$

8 Para un mayor detalle de las mismas, puede revisar Aroca (2001) o, Soza (2008 ó 2007). 
Donde:

n : Corresponde al número de sectores

A : Matriz de coeficientes técnicos, que se descompone en las siguientes submatrices:

$\mathbf{A}_{11} \quad \mathbf{A}_{12}$

$\mathbf{A}_{21} \quad \mathbf{A}_{22}$

i : vector fila (unitario)

$\mathrm{t} \quad$ : Indica que un vector o matriz está transpuesto

$\overrightarrow{\mathbf{B}}$ : Matriz inversa de distribución, que se descompone como sigue:

$\overrightarrow{\mathbf{B}}_{11} \quad \overrightarrow{\mathbf{B}}_{12}$

$\overrightarrow{\mathbf{B}}_{21} \quad \overrightarrow{\mathbf{B}}_{22}$

$\mathbf{x} \quad$ : Output (producción total)

y : Demanda final (consumo (privado y del gobierno), formación bruta de capital y exportaciones)

r : Inputs Primarios

$\mathbf{w}$ : Salarios

$\mathbf{G}_{22}:\left(I-A_{22}\right)^{-1}$

$\mathbf{p}$ : Ponderador, $\mathbf{X i}$ / $\mathbf{x}$; para una economía desarrollada y; $\mathbf{Y i}$ / $\mathbf{y}$; para una en vías de desarrollo

$\mathbf{W}_{22}:\left(\mathbf{i}-\overrightarrow{\mathbf{B}}_{22}\right)^{-1}$

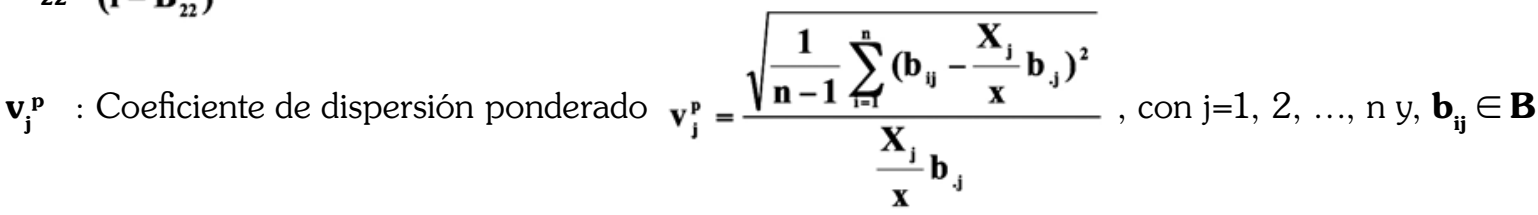

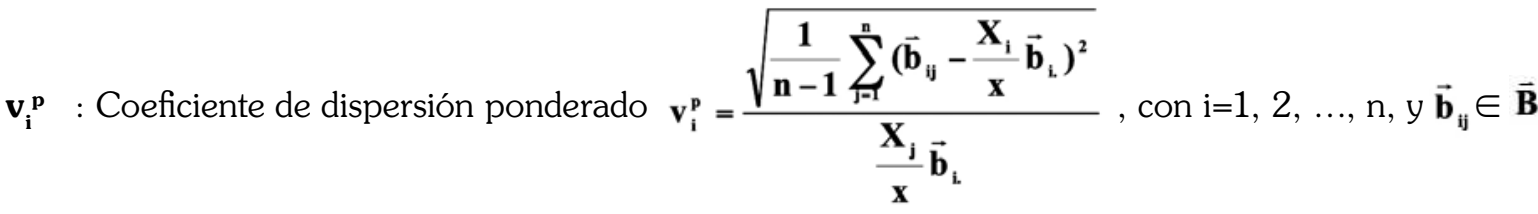

Tipo de sector según su encadenamiento después de relativizar

\begin{tabular}{|c|c|c|}
\hline & BL $>\mathbf{1}$ & BL $<\mathbf{1}$ \\
\hline $\mathbf{F L}>\mathbf{1}$ & Clave & Base \\
\hline $\mathbf{F L}<\mathbf{1}$ & Impulsor & Enclave o Isla \\
\hline
\end{tabular}




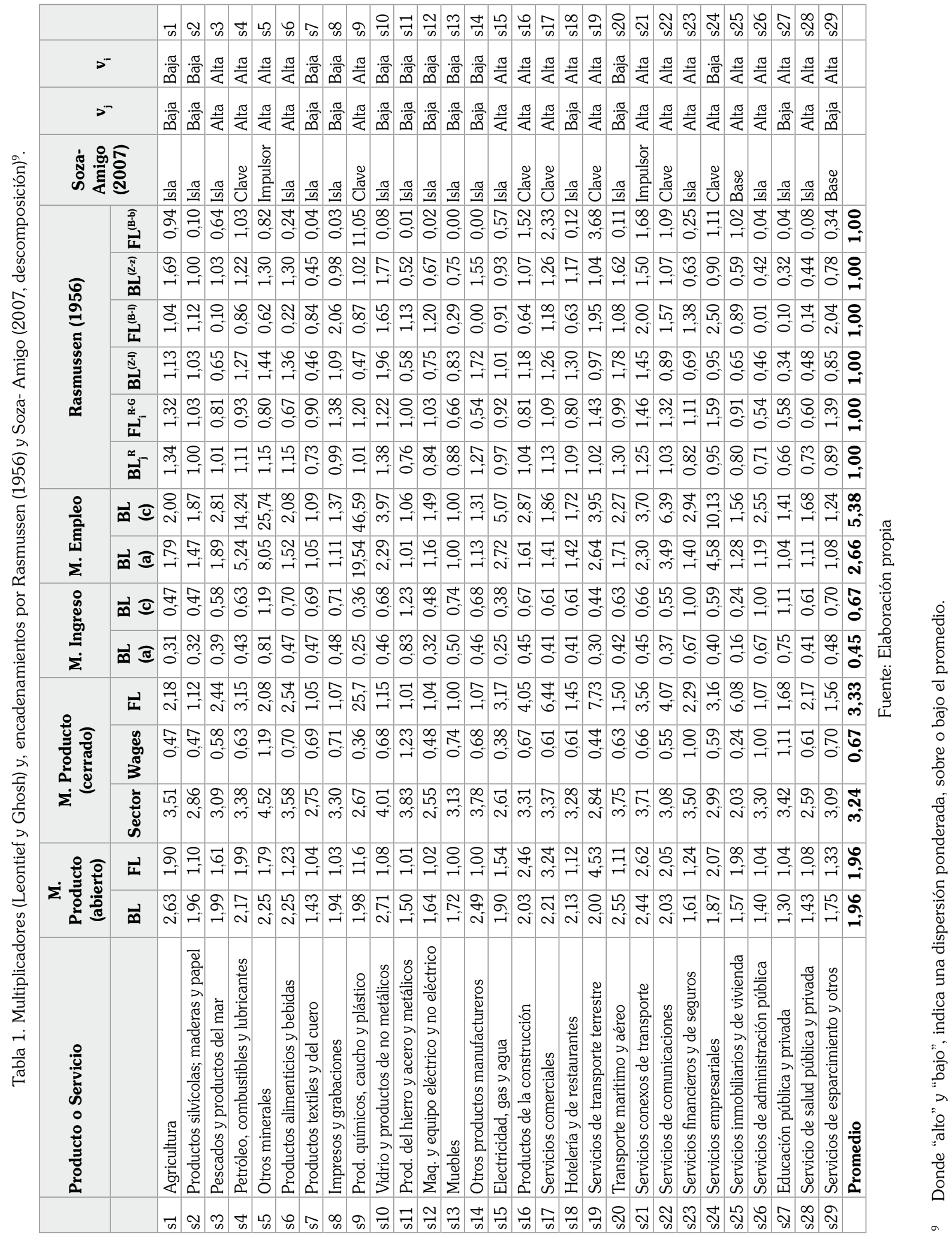




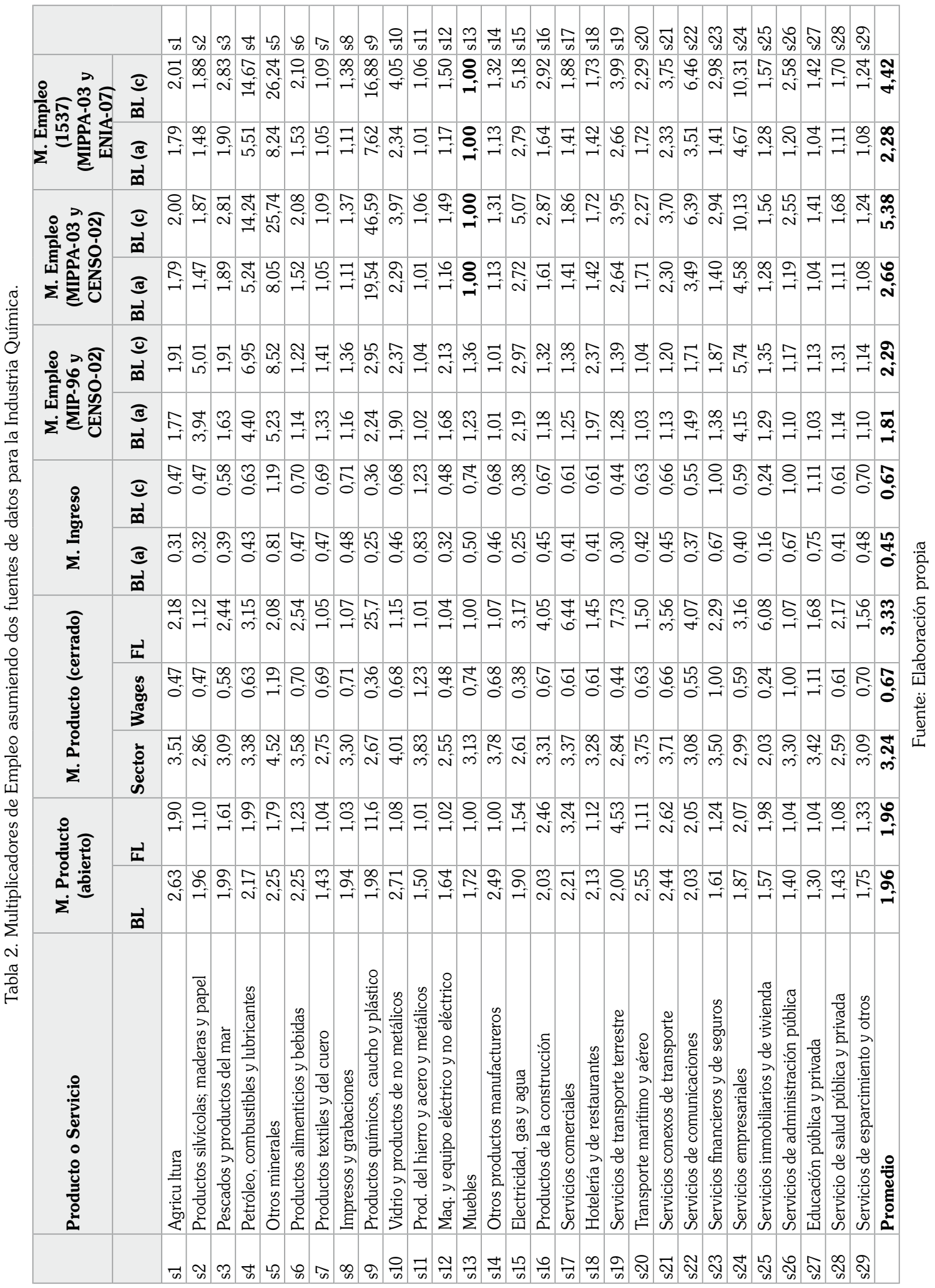


